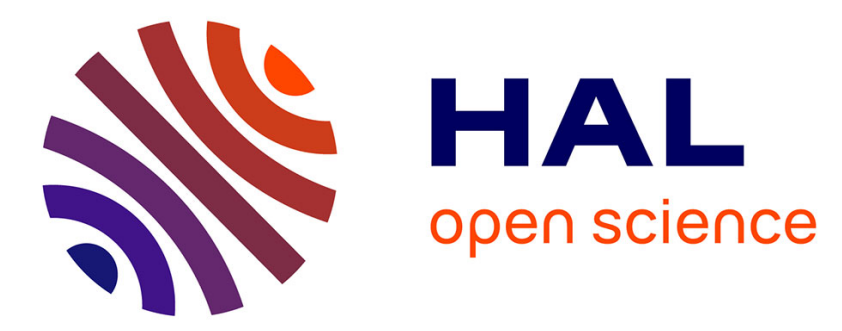

\title{
Transient destabilisation in anisotropic vibrational properties of wood when changing humidity
}

\author{
Iris Brémaud, Joseph Gril
}

\section{To cite this version:}

Iris Brémaud, Joseph Gril. Transient destabilisation in anisotropic vibrational properties of wood when changing humidity. Holzforschung, 2021, 75 (4), pp.328-344. 10.1515/hf-2020-0029 . hal-03013259

\section{HAL Id: hal-03013259 \\ https://hal.science/hal-03013259}

Submitted on 18 Nov 2020

HAL is a multi-disciplinary open access archive for the deposit and dissemination of scientific research documents, whether they are published or not. The documents may come from teaching and research institutions in France or abroad, or from public or private research centers.
L'archive ouverte pluridisciplinaire HAL, est destinée au dépôt et à la diffusion de documents scientifiques de niveau recherche, publiés ou non, émanant des établissements d'enseignement et de recherche français ou étrangers, des laboratoires publics ou privés. 


\section{Original article}

Iris Brémaud* and Joseph Gril

\section{Transient destabilisation in anisotropic vibrational properties of wood when changing humidity}

https://doi.org/10.1515/hf-2020-0029

Received January 23, 2020; accepted July 17, 2020; published online September 25, 2020

Abstract: Moisture changes cause transient effects in various polymeric materials. In wood, they are mainly documented in semi-static or low-frequency domains and could explain various anomalies in wood behaviour, including the so-called mechano-sorptive effect. This article aimed at exploring and quantifying transient effects in wood vibrational properties, evidenced but in few publications. A series of 65 experiments on spruce and maple, in longitudinal (L) and radial (R) directions, spanning many humidity trajectories, were monitored through time (1-7 weeks) after step-change in relative humidity (RH). Changes in dimensions and specific dynamic modulus of elasticity $\left(E^{\prime} / y\right)$ closely followed change in moisture content $(\mathrm{MC})$. But the damping coefficient $(\tan \delta)$ always increased (whether MC increased or decreased) within first hours/days, before slowly re-decreasing. This was quantitatively analysed by subtracting equilibrium moisture content (EMC) dependence from the global changes in $\tan \delta$, which simultaneously expressed destabilisation, followed by physical ageing (a slow process towards approaching equilibrium). For small EMC steps, the amplitude of destabilisation in $\mathrm{L} \tan \delta$ exceeded changes due to EMC dependence. Destabilisation was of similar importance relative to $\mathrm{L}$ or to $\mathrm{R} \tan \delta$, while $\mathrm{R} \tan \delta$ showed much (2-4X) stronger EMC dependence. Amplitude of destabilisation increased with wider RH/EMC-steps (and faster sorption rates). Within an experiment, maximum destabilisation occurred nearly concomitantly with maximum sorption rate. Equilibrium was usually reached

\footnotetext{
*Corresponding author: Iris Brémaud, Wood Team, LMGC, CNRS, Université de Montpellier, Montpellier, F34090, France, E-mail: iris.bremaud@cnrs.fr. https://orcid.org/0000-0001-7999-6661 Joseph Gril, Institut Pascal, CNRS, Université Clermont Auvergne, Sigma Clermont, F63000, Clermont-Ferrand, France; and PIAF, Université Clermont Auvergne, INRAE, F63000, Clermont-Ferrand, France
}

in MC, even within one week, but seldom in $\tan \delta$, even after several weeks. Results suggested that: (i) drying caused similar amplitudes of destabilisation, but that occurred sooner, and recovered faster, than humidifying; (ii) small RH-steps caused relatively smaller amplitudes of destabilisation, but that occurred later, and spanned over longer time before recovering equilibrium, than wide RH-steps. Some possible hypotheses are introduced to explain the observed phenomena. These phenomena also have important practical consequences in real-life wood uses and, particularly, in the design of experimental protocols.

Keywords: damping by internal friction; destabilisation by moisture sorption; dynamic mechanical properties; physical ageing; transient effects; viscoelastic vibrational properties.

This work is dedicated to the memory of Prof. David G. Hunt, as homage to his pioneering research on wood rheology, including transient effects and physical ageing in wood.

\section{Introduction}

Wood being a hygroscopic material, its physical and mechanical behaviour is strongly influenced by humidity. One part of this is the dependence of properties on internal equilibrium moisture content (EMC) of wood, where equilibrium could be defined as the stable moisture content reached after sufficient storage in a given, controlled condition of relative humidity (RH). However, real life is seldom at equilibrium.

Changes in humidity cause transient effects in physical and mechanical properties on a variety of semi-amorphous polymeric materials, either natural or artificial, that have important consequences in several fields of uses (Back et al. 1983; Wang et al.1991). Such transient effects are very important in wood science. Changing moisture causes instability that may accelerate the normal time-dependent behaviour of wood (Hunt and Gril 1996; Ishimaru et al. 2001). This has strong practical consequences, and is 
investigated, in the fields notably of mechano-sorptive creep and relaxation (Armstrong and Kingston 1960; Back et al. 1983; Engelund and Salmén 2012; Gril 1988; Hunt 1997; Ishimaru et al. 2001; Montero et al. 2012; Navi et al. 2002; Peng et al. 2019; Ranta-Maunus 1975; Takahashi et al. 2004, 2005, 2006), but also of dimensional changes/ shrinkage (Hunt 1990, 1997), and in dynamic mechanical analysis at low frequencies (Ebrahimzadeh 1997; Ebrahimzadeh and Kubät 1993; Salmén and Olsson 2016; Zhan et al. 2016; Zhan et al. 2018a,b).

Transient effects of changing humidity are also evidenced in the audio-frequency domain, i.e. viscoelastic vibrational properties, by a small number of works (Gril 1988; Hunt 1997; Hunt and Gril 1996; Suzuki 1962; Sasaki et al. 1988). Vibrational properties include specific dynamic modulus of elasticity $\left(E^{\prime} / \gamma\right.$, where $E^{\prime}$ is the storage modulus and $y$ is the - dimensionless - specific gravity) and damping coefficient $(\tan \delta)$ which represents internal friction or loss factor (Obataya et al. 2000). They are a very useful means to study wood viscoelastic behaviour, because their measurement can be very rapid and reproducible, i.e. allows to study fine kinetics and to compare many tests, and their analysis can provide insights on microstructural and/or physico-chemical sources of variability (Brémaud et al. 2013; Norimoto et al. 1986; Obataya et al. 1998; Obataya et al. 2000).

Humidity changes induce slight transient changes in $E^{\prime} / \gamma$ (Hunt 1997; Sasaki et al. 1988), and strong ones in $\tan \delta$. Typically, $\tan \delta$ shows a rapid transient increase after changing humidity conditions, whether in drying or in humidifying, before slowly re-decreasing during time of storage in the new condition (Gril 1988; Hunt 1997; Hunt and Gril 1996; Suzuki 1962; Sasaki et al. 1988). Hunt and Gril (1996) describe this as destabilisation followed by physical ageing, i.e. "timedependent approach of a polymer to thermodynamic equilibrium”. In various polymeric materials, changing humidity (or temperature) is thought to cause a rejuvenation of the material, that will then age again (Struik 1977). There are only few studies of these phenomena for wood, yet they suggest (in creep/relaxation tests) that changes in humidity create an unstable state that increases fluidity (Ishimaru et al. 2001) and this depends on specimen's history, i.e. a kind of memory effect (Takahashi et al. 2006). Following a destabilisation, the approach towards new equilibrium, i.e. physical ageing, is found to be very slow (Ishimaru et al. 2001) or even, in green wood, seemingly "never ending” (Dlouha et al. 2009; Obataya et al. 2020). Hunt and Gril (1996) put forward the hypothesis that destabilisation followed by physical ageing can explain various observed anomalies in wood behaviour.

These transient effects, due to the changes in moisture, are superimposed to the effect of the moisture content (MC) itself on physical-mechanical properties, that can be expressed by their EMC dependence, once that equilibrium is reached. For wood vibrational properties, $E^{\prime} / y$ decreases, and $\tan \delta$ increases, with increasing EMC (Obataya et al. 1998), and the degree of EMC dependence is different in the longitudinal (L) and radial (R) directions of wood (Brémaud and Gril 2020). In addition, transient effects of changing moisture can concern, simultaneously, different domains of wood behaviour, e.g. dimensional changes/ shrinkage, creep, and vibrational properties (Hunt 1997). Furthermore, beyond changes in material properties themselves, changes in humidity can involve transient moisture gradients within a piece of wood, that can induce internal stresses (Konopka and Kaliske 2018). Therefore, changing humidity has both important, and complex, practical consequences for wooden objects or structures, both newly-built or belonging to tangible cultural heritage. For example in a musical instrument facing an environment with changing humidity, there might be structural risks due to transient effects on dimensional changes and shrinkage/swelling stresses, and to mechano-sorptive creep under the loading of the strings (Fioravanti et al. 2013; Goli et al. 2012), while variations in "sound quality" could also be clearly perceptible by listeners (Borland 2014; Torres et al. 2014). In scientific research on wood, these cumulated (transient and EMC) effects of humidity/moisture also mean that experimental protocols should be carefully designed in order to discriminate between the various intertwined phenomena involved.

Existing literature on the transient effects of moisture changes on vibrational properties highlights their importance and outline physical hypothesis on the nature of the observed phenomena (Gril 1988; Hunt 1997; Hunt and Gril 1996; Sasaki et al. 1988; Suzuki 1962). However, the transient effects are usually not separated from the EMC dependence; the number and range of humidity steps is often limited (only wide, or only small, RH steps, or wide and small steps but only in humidifying); and up to now mostly softwoods in $\mathrm{L}$ direction have been tested.

Hence the purpose of the present research was to better document an array of factors that might influence the transient effects on vibrational properties. A series of experiments of monitoring changes in moisture and vibrational properties through time after step-change in conditions was undertaken, covering a wide span of humidity steps in ad- and de-sorption, measured in $\mathrm{L}$ and $\mathrm{R}$ directions, on a softwood (spruce) and a hardwood (maple). The quasi-equilibrium moisture dependence, explored in a related article by the authors (Brémaud and Gril 2020), was subtracted from transient changes, and some data analysis methods were proposed to quantify 
both the amplitude, and time of occurrence, of maximum destabilisation, and to evaluate if properties were approaching an "equilibrium" through the slow process of physical ageing.

\section{Materials and methods}

\subsection{Wood material and specimen preparation}

The study was based on Norway spruce (Picea abies [L.] Karst.) as a softwood, and sycamore maple (Acer pseudoplatanus L.) as a hardwood. Material was selected for being straight grain and homogeneous. Specimens with dimensions of $120 \times 2 \times 12 \mathrm{~mm}^{3}(\mathrm{R} \times \mathrm{T} \times \mathrm{L})$ for properties in radial (R) direction, of $12 \times 2 \times 150 \mathrm{~mm}^{3}(\mathrm{R} \times \mathrm{T} \times \mathrm{L})$ for properties in longitudinal (L) direction, were machined with a very fine planed surface. Dimensions were a trade-off: sufficiently small to minimise gradients of moisture diffusion, sufficiently large for very smooth surface machining and for precise measurements of dimensional changes. Care was taken to reduce variability: material came from medium heartwood and series of specimens were matched by cutting them in sequences following material axes. Matched series of $L$ specimens were aligned between them along $\mathrm{T}$ or $\mathrm{L}$ direction, and matched series of $\mathrm{R}$ specimens were aligned along $\mathrm{L}$ direction, i.e. a matched series of specimens always came from a same group of annual rings. After initial vibrational measurements, the actual similarity between specimens was checked. The annual ring width was of $1-2.5 \mathrm{~mm}$ for both spruce and maple. The specific gravity ( $\gamma$, unitless) was of $0.47 \pm 0.02$ for spruce and $0.63 \pm 0.03$ for maple.

\subsection{Vibrational measurements and quantification of errors}

Tests were conducted by non-contact forced vibrations of free-free slender beams (Fukada 1950; Obataya et al. 2000), using a custombuilt hardware and software device (Brémaud 2006; Brémaud et al. 2012). Specific dynamic modulus of elasticity $\left(E^{\prime} / y\right)$ was calculated from the resonance frequency at the 1st mode of flexural vibration according to Euler-Bernoulli equation. Resonance frequencies were in the range of $100-300 \mathrm{~Hz}$ for $\mathrm{R}$ specimens and of $500-700 \mathrm{~Hz}$ for $\mathrm{L}$ specimens. Vibrational properties are relatively independent of frequency in the range 100-2000 $\mathrm{Hz}$ (Ono and Kataoka 1979). Viscoelastic damping coefficient $\tan \delta$ ( $\approx$ internal friction or loss factor) was obtained in the frequency domain by the bandwidth method ( $\tan \delta=Q^{-1}$ with $Q$ being “quality factor”, as illustrated in Brémaud et al. 2012).

The possible sources of errors, with this actual device and setup, have been quantified (Brémaud et al. 2012; Brémaud and Gril 2020). Experimental errors in $E^{\prime} / y$ due to frequencies were negligibly small, but those due to thickness could have a noticeable effect, despite precise machining. Thus, after initially recording the homogeneity over specimens, dimensions were always measured at a same position for the monitoring of changes through time. The precision of measurements of $\tan \delta$ by bandwidth increases with acquisition time (Brémaud et al. 2012, Brémaud and Gril 2020). Here, an acquisition time of $20 \mathrm{~s}$ allowed fine monitoring of immediate changes through time, with a resolution $\leq 1.5 \%$ (i.e. testing resolution of 0.00007 compared to 0.0065 average "air-dry" nominal value of $\tan \delta_{\mathrm{L}}$, of 0.00019 compared to 0.021 for $\tan \delta_{\mathrm{R}}$ ). Relative errors between 10 repetitions gave a coefficient of variation (standard deviation/ average) $\leq 1 \%$ and a range of variation ([maximum - minimum]/ average) $\leq 3 \%$ for both $\mathrm{R}$ and $\mathrm{L} \tan \delta$. Specimens were manipulated with tweezers instead of fingers, and vibration tests for monitoring through time were conducted inside a climatic equipment (Brémaud and Gril 2020).

\subsection{Trajectories of humidity changes}

The whole wood material had been naturally air-dried for at least one year before sampling and machining. Then specimens were stabilised for more than one month at $20{ }^{\circ} \mathrm{C}$ and $65 \% \mathrm{RH}$, before initial measurements of mass, dimensions and vibrational properties. The main sampling and protocol (H for "Humidity Pathways", Figure 1) started from this condition. It consisted of a matched series of $18 \mathrm{~L}$ and $18 \mathrm{R}$ specimens for each of the two species. Three groups were brought, and three pairs of specimens were monitored through time, from $65 \% \mathrm{RH}$ to 35,50 and $85 \% \mathrm{RH}$; then groups were re-divided into pairs to monitor changes from these conditions to 35, 50, 65 and 85\% RH. Additional experiments came from previous protocols (Brémaud and Gril 2020) mostly studied for EMC dependence on groups of specimens (plain straight arrows in Figure 1) with part of their steps also studied for monitoring (dashed or dotted curved arrows in Figure 1). A and D samplings used matched series of specimens very similar to those of $\mathrm{H}$ sampling. Protocol A ("full Adsorption" from oven-dry state) and protocol D ("full Desorption” from water-saturated) each included three monitoring experiments, over one pair of specimens per species and per direction (total of four pairs). Protocol V ("Variability and cycles”, spruce only) started from the same conditions as protocol $\mathrm{H}$ (never oven-dried nor re-saturated) and included three monitoring experiments, over two pairs of specimens per direction. Because the $\mathrm{V}$ sampling involved natural material variability, the respective two paths for the two pairs are shown in Figure 1.

A short coding for protocols and sampling followed the general scheme: "Species Direction Protocol", where S = Spruce, M = Maple; $\mathrm{L}=$ Longitudinal, $\mathrm{R}$ = Radial; Protocol H, A, D, or V. For example: $\mathrm{SLH}=$ Spruce, Longitudinal, $\mathrm{H}$ protocol; or MRA = Maple, Radial, A protocol.

\subsection{Monitoring of changes in properties through time}

For each combination of initial and final humidity conditions, the (quasi-)equilibrium value of initial properties was first measured, usually inside the climatic equipment for initial condition, or, occasionally, within $\leq 2 \mathrm{~min}$ outside. When leaving the initial condition, specimens were placed in an air-tight sealed plastic bag, brought to the next condition (time lag $\leq 2 \mathrm{~min}$ ), and the bag was opened just before starting the monitoring of changes in properties through time in the new controlled condition. For each species and anisotropic direction, two paired specimens were used, one for monitoring changes in vibrational properties, one for monitoring changes in mass and dimensions. The matching procedure insured that differences in initial properties between two paired specimens for a test was $\leq 2 \%$ (recorded differences in EMC, $\gamma, E^{\prime} / \gamma$, $\tan \delta$, stabilised for 1 month at $20^{\circ} \mathrm{C}$ and $65 \% \mathrm{RH})$. Paired specimens were used to avoid constantly manipulating specimens between the devices for vibrational, weight and 


\begin{tabular}{|c|c|c|c|}
\hline \multirow{3}{*}{ Protocol: } & \multicolumn{3}{|c|}{ Sampling for each trajectory of humidity changes } \\
\hline & H & A & V \\
\hline & Spruce + Maple & Spruce + Maple & Spruce \\
\hline Specimens for group EMC & - & $(8 \mathrm{~L}+8 \mathrm{R}) \times 2 \quad(8 \mathrm{~L}+8 \mathrm{R}) \times 2$ & $40 \mathrm{~L}+12 \mathrm{R}$ \\
\hline \multirow{2}{*}{$\begin{array}{l}\text { Specimens for transients } \\
\text { Monitoring experiments }\end{array}$} & $(18 \mathrm{~L}+18 \mathrm{R}) \times 2$ & $(2 L+2 R) \times 2 \quad(2 L+2 R) \times 2$ & $4 \mathrm{~L}+4 \mathrm{R}$ \\
\hline & 40 & 11 & 6 \\
\hline \multicolumn{4}{|l|}{$\underline{\text { Conditions }}$} \\
\hline \multicolumn{4}{|l|}{$85 \%$ RH } \\
\hline \multicolumn{4}{|l|}{$65 \% \mathrm{RH}$} \\
\hline \multicolumn{4}{|l|}{$50 \% \mathrm{RH}$} \\
\hline \multicolumn{4}{|l|}{$35 \% \mathrm{RH}$} \\
\hline Dessicator & & & \\
\hline Oven-dry & U. & 1 & 光 \\
\hline
\end{tabular}

Figure 1: Protocols and samplings for trajectories of changes in humidity. Numbers: order of successive humidity conditions. Curved dotted or dashed arrows: monitoring through time the changes in properties after step-change in conditions (same colour = same pair of specimens); straight arrows: trajectories for studying EMC dependence (Brémaud and Gril 2020). Circles: measurement of mass, dimensions and vibrational properties at quasi-equilibrium (open circles: on a pair of specimens for studying transients; filled circles: on a full group of specimens to study EMC dependence). Squares: measurement of oven-dry mass and dimensions. to interpolate the experimental data to obtain all parameters at the same times.

An interpolation procedure was applied to all experimental curves of changes in properties through time, using a custom-made algorithm developed with Visual Basics ${ }^{\circledR}$. Curves of changes in properties were plotted in a common logarithmic (base 10) scale of time (expressed in minutes for convenience) and were locally fitted to a second-order polynomial using a Gaussian kernel for weighting data. The standard deviation of the Gaussian kernel expresses the width of the window on which the local polynomial interpolation is done. A constant criterion was used for this parameter, $\Delta \log t(\min )=1$. This value is a trade-off to smooth discontinuities, with only very slight flattening of curves (Figure 2). Throughout this article, log means $\log 10$, i.e. decimal or common logarithm. The interval between interpolated values $\log t(\mathrm{~min})=0.25$ was used. This procedure also allowed to smooth experimental noise. At the same time, the derivative $\mathrm{d}$ Property/dlogt was computed (for example, the 1st derivative of mass changes over log-time, $\mathrm{d} m / \mathrm{d} \log t$, will describe sorption rate in the discussion). Interpolated data allowed to combine the experimental data from vibrational (damping and resonance frequency) and physical (mass and dimensions) tests obtained from two paired specimens at distinct times. This allowed to strictly compare the changes over time in parameters (open symbols in Figure 3) calculated from a pair of specimens, such as changes in MC and in specific modulus $E^{\prime} / \gamma$, at the same interval as parameters (filled symbols in Figure 3) obtained from a single specimen such as changes in damping $\tan \delta$.

\section{Results and discussion} slowed down the timescale of monitoring. During the very beginning of the transition, measurements points were conducted every minute, then every few minutes. Points of measurements were gradually more and more distant during the first day, then every few days during the 1st week, then monitoring continued to be done occasionally for 2.5 weeks in average (from 1 to 7 weeks). A monitoring experiment was defined as studied on: a pair of specimens $\times$ a species $\times$ a direction $\times$ a sampling/protocol $\times$ a given humidity step. All steps identified by curved dotted/dashed arrows in Figure 1 indicate monitoring experiments. All of these were studied for spruce, in L direction, and in the vast majority also in R direction and on maple. Occasionally, a small number of $\mathrm{R}$ or maple experiments were missing due to practical circumstances (for example, protocol "A" included 11 monitoring experiments instead of the intended 12). The total number of monitoring experiments was of 65 . They were compared to values at quasiequilibrium as measured on a full group of specimens after at least 23 weeks in controlled conditions (Brémaud and Gril 2020). The average number of measurement points per monitoring experiment was of 52 (but ranging from 24 to 191), giving a total number of 3312 measurement points for vibrational properties monitoring through time (plus 1018 measurement points for quasi-equilibrium MC dependence (Brémaud and Gril 2020)).

\subsection{Post-treatment of data}

Experimental data of monitoring through time included slight timeinterval differences between two paired specimens (vibrational and physical measurements) for a given experiment, and wide timeinterval differences between different experiments. It was thus needed

\subsection{Changes in properties through time after changing RH conditions}

Figure 3 presents two typical examples of monitoring changes in properties through time after a step change in $\mathrm{RH}$ : one in adsorption, on specimens previously stabilised at $35 \% \mathrm{RH}$, and immediately placed at $65 \% \mathrm{RH}$; the other in desorption, between the same two RH. During the first hours after changing conditions, the MC changed very fast. On these dimensions, the increment in MC ( $\triangle M C$ in Figure 3) reached $50 \%$ of its total changes after, in average, approx. $9 \mathrm{~h}$ (L specimens) or $6 \mathrm{~h}(\mathrm{R})$ in adsorption, and after only $1.3 \mathrm{~h}(\mathrm{~L})$ or $0.6 \mathrm{~h}(\mathrm{R})$ in desorption. After $24 \mathrm{~h}$, it usually reached about $80 \%$ (in adsorption) or $90 \%$ (in desorption) of its total change. The relative variations in dimensions ( $\mathrm{d} w$ and $\mathrm{d} h$ in Figure 3), resonance frequency (dfr), and therefore specific modulus of elasticity $\left(\mathrm{d} E^{\prime} / \gamma\right)$, changed accordingly, in a close to linear relation with $\triangle M C$ (Figure 3 lower row). But damping $(\tan \delta)$ always increased in the first hours after changing conditions, whether the moisture decreased or increased. Then it decreased again very gradually towards its value in the new condition, a stabilisation which occurred long after the mass of the specimens could appear stable. The values of $\tan \delta$ could be 


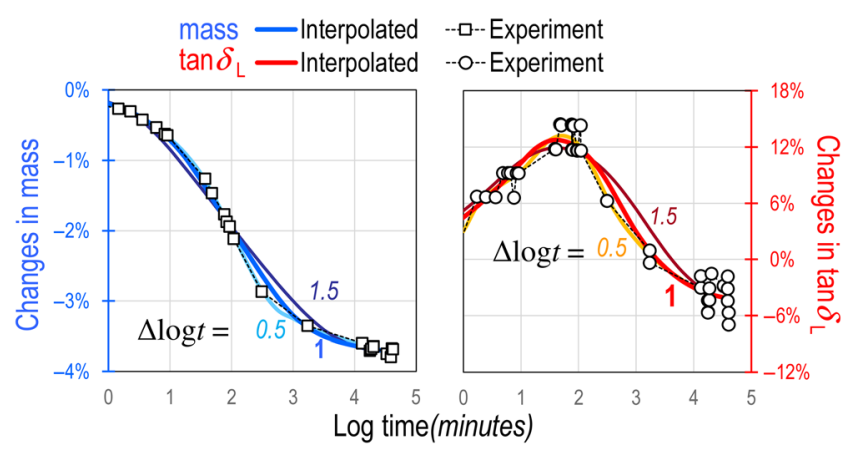

Figure 2: Smoothing and interpolation of experimental data, illustrated for changes in mass (left) and in damping coefficient tan $\delta_{\mathrm{L}}$ (right) through time. Interpolation is shown for different values of the standard deviation of the Gaussian kernel ( $\Delta$ logt). The value chosen for the analysis $(\Delta \log t=1)$ is a trade-off to smooth experimental noise with only slight flattening of curves. Example: from 65 to $35 \% \mathrm{RH}$, protocol $\mathrm{H}$, spruce in $\mathrm{L}$ direction.

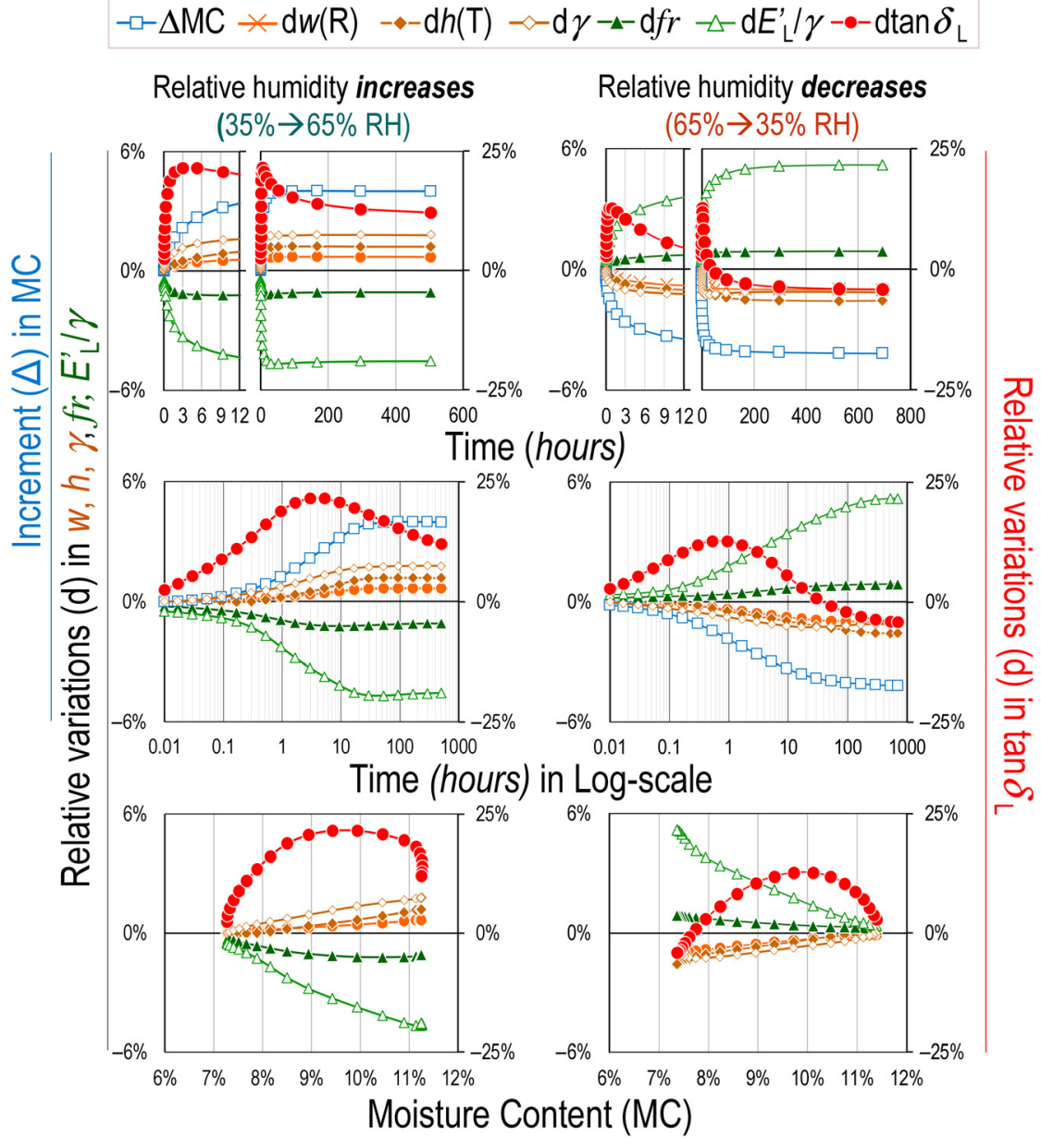

Figure 3: Monitoring of changes in properties through time after a stepchange in RH. Examples between 35 and $65 \% \mathrm{RH}$, protocol $\mathrm{H}$, spruce in $\mathrm{L}$ direction. Left: adsorption, right: desorption. Upper row: linear scale of time - an insert is made on the initial few hours. Middle row: logarithmic scale of time. Lower row: changes in physical-mechanical properties plotted against changes in MC. Changes are shown as increment $(\Delta)$ for $M C$, and as percent variations $(d)$ relative to initial state for all other properties. Filled symbols: direct experimental data on a single specimen (damping coefficient: $\tan \delta$; resonance frequency: $f r$, width/R dimension: $w(R)$; thickness/T dimension $h(\mathrm{~T})$ ); open symbols: data from parallel monitoring of two paired specimens (specific gravity: $\gamma$, specific dynamic modulus of elasticity $E^{\prime} / \gamma$ ). related to those in $\mathrm{MC}$ at the initial and final states, but they could not be related to MC during the changes through time (Figure 3 lower row).

For experimenters, it means that characterisations of damping should absolutely be conducted in controlled conditions, and after a sufficiently long time in such conditions, i.e. a specimen considered as "stabilised" on the basis of its changes in mass, or in other physical-mechanical properties, is not necessarily so regarding damping.

The same trend of change in $\tan \delta$, i.e. rapid transient increase followed by slow re-decrease, was observed in all 65 conducted experiments (Figure 4). The maximum of 
$\tan \delta$ occurred between the first half-an-hour up to the first two days after changing RH conditions. In ad-sorption, it occurred after $18 \mathrm{~h}$ in average (4-53 h), and in de-sorption after $1 \mathrm{~h}$ in average $(5 \mathrm{~min}-4 \mathrm{~h})$. For protocols involving near-anhydrous or water-saturated states (not shown in Figure 4) the maximum occurred very soon, from 3 to 20 min after changing condition. Between different RH, maximum $\tan \delta$ seemed to occur latest, i.e. the destabilisation seemed to span over longest time, for the smallest steps of RH. In ad-sorption, maximum $\tan \delta$ occurred twice later for $\mathrm{R}$ specimens ( $24 \mathrm{~h}$ in average, 4-53 h) than for $\mathrm{L}$ ones (11 $\mathrm{h}$ in average, 4-30 h). On the contrary, in desorption, it occurred twice sooner for R specimens $(0.5 \mathrm{~h}$ in average, $5 \mathrm{~min}-2 \mathrm{~h}$ ) than for $\mathrm{L}$ ones $(1.3 \mathrm{~h}$ in average, $5 \mathrm{~min}-4 \mathrm{~h}$ ). These indicative times are of practical significance, for real life use and for experimenters. However, as such they are difficult to analyse physically. For instance, the delayed occurrence of maximum $\tan \delta$ for $\mathrm{R}$ specimens in adsorption is counter-intuitive, given that their dimensions and orientations involve a somewhat faster moistening, especially in the beginning. Also, in desorption (Figure 4c, d), the destabilisation competes with the drying effect, resulting in apparently shorter timescale, and apparently smaller amplitude, for the peak in $\tan \delta$. The different phases of the overall process should be separated to be analysed.

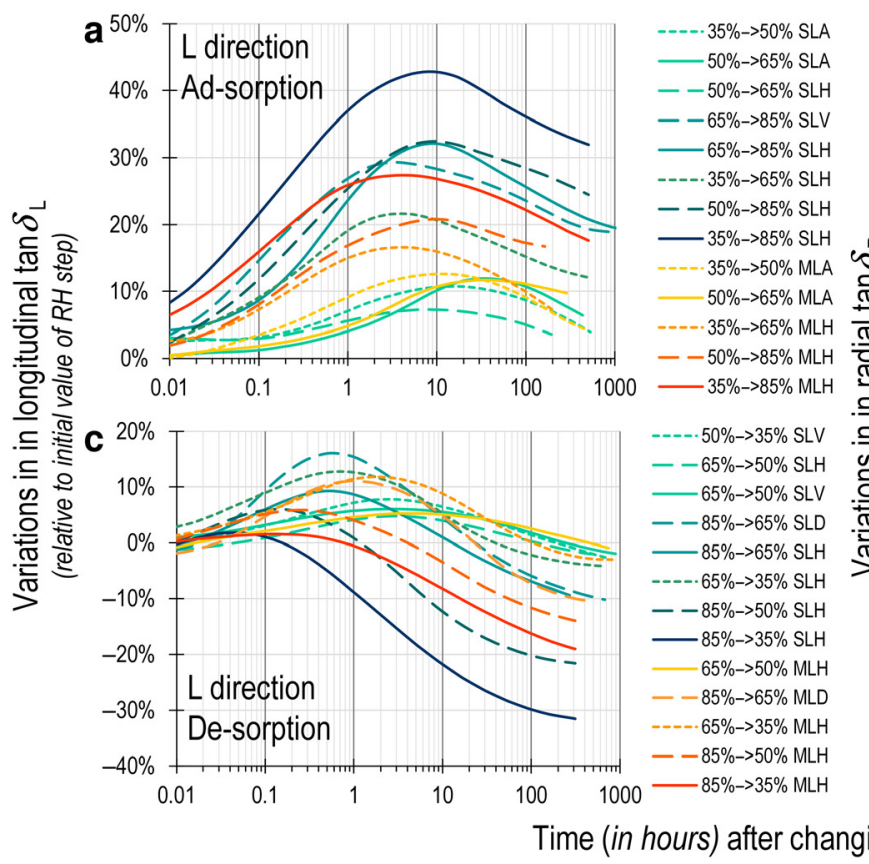

These transient, non-equilibrium changes in damping express distinct but superimposed phenomena. Given the small cross-section of specimens, the diffusion process inside cell walls should predominate over that inside the specimen. Although potential effects of moisture gradients inside specimens cannot be completely ruled out, and will be shortly discussed in following sections, the main explanatory phenomena lie at ultrastructural and molecular scales. First, the transient strong increase in $\tan \delta$, corresponds to a destabilisation effect (Gril 1988; Sasaki et al. 1988; Suzuki 1962). Concomitantly, the effect of drying or moistening modifies $\tan \delta$, in a way that should correspond to what is known for EMC dependence (Brémaud and Gril 2020; Obataya et al. 1998). Then, the gradual decrease over time, towards equilibrium values, corresponds to physical ageing, i.e. to slow approach to an asymptotical value following a "rejuvenation" caused by change in humidity (Dlouha et al. 2009; Hunt and Gril 1996; Struik 1977). The global experimental curves could be described by characteristic times for the different stages of evolution (Hunt 1997), however destabilisation and stabilisation occur simultaneously, both in drying and in humidifying (Takahashi et al. 2004, 2005). The following sections of this article will aim to separate and quantify the competing effects of destabilisation, and of MC dependence.

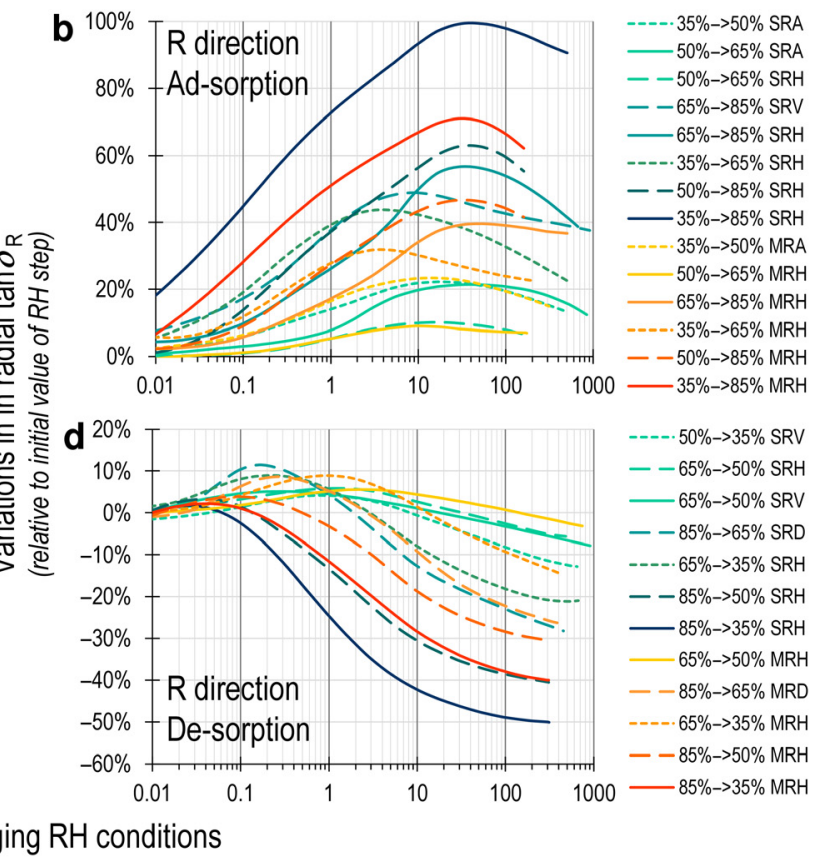

Figure 4: Variations (relative to value in initial condition) in damping coefficient tan $\delta$ through time after step-change in RH, for all tested samples and RH-steps. (a, b) ad-sorption, (c, d) de-sorption, (a, c) longitudinal (L) and (b, d) radial (R) directions. RH steps, species, material direction, and protocol (Figure 1) are indicated in the legend. Turquoise-to-blue curves: spruce, yellow-to-red curves: maple. 


\subsection{Transient effects versus EMC dependence for damping and specific dynamic modulus of elasticity}

A first approach to visualise the respective effects of transient destabilisation, and of MC dependence, is shown in Figure 5. The differences between the initial and final points corresponded to what was expected for moisture dependence at quasi-equilibrium (Brémaud and Gril 2020; Obataya et al. 1998). In between, the transient values of $\tan \delta$ (Figure $5 \mathrm{a}$ ) were always strongly greater than what would have been expected through a virtual linear relation between $\tan \delta$ and MC. Furthermore, for small RH steps (e.g. between 35 and $50 \% \mathrm{RH}$, or between 50 and $65 \% \mathrm{RH}$ ), the

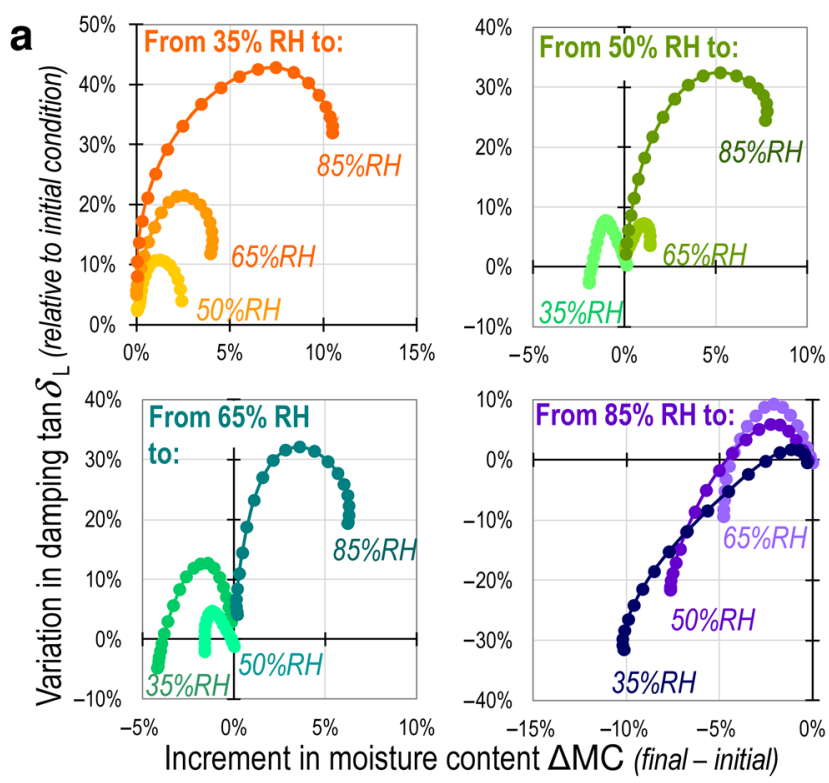

b

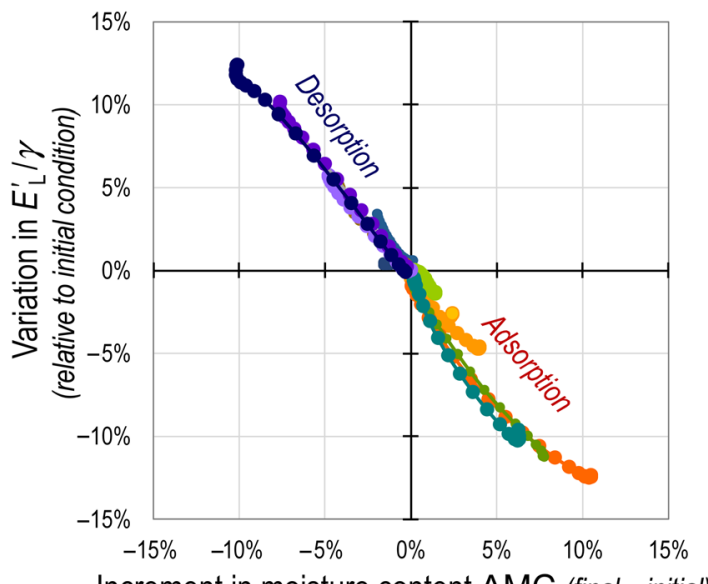

Increment in moisture content $\Delta \mathrm{MC}$ (final - initial)

Figure 5: Variations (relative to initial value, after a step-change in $\mathrm{RH}$ ) in (a) damping $\tan \delta$ and (b) specific modulus of elasticity $E^{\prime} / \gamma$, for spruce in longitudinal direction, as a function of increment in MC. transient changes in damping clearly exceeded the differences between initial and final values.

The relative variations in specific modulus (Figure $5 b$ ) showed much less destabilisation, although there were neither strictly linear along MC changes. On a wide step (35-85\% RH), the change in $E^{\prime} / \gamma$ due to destabilisation (i.e. non-linear part) amounted to $-2.5 \%$ compared with a final increment of $-12.5 \%$, that is, a ratio of $1 / 5$ whereas for $\tan \delta$ this ratio was of nearly $1 / 1$. A greater sensitivity of the viscoelastic, than the elastic, behaviour to destabilisation is evidenced in creep or relaxation tests (Engelund and Salmén 2012; Ishimaru et al. 2001; Peng et al. 2019). In the dynamic range, $\tan \delta$ is theoretically much more sensitive to physicochemical variations, given that it reflects the behaviour of the amorphous matrix, whereas $E^{\prime} / y$ is governed by microfibrils of crystalline cellulose (Norimoto et al. 1986). Part of the transient in $E^{\prime} / \gamma$ might also express moisture gradients through the specimen thickness, the outer layer (important in flexural vibrations) being moisture-softened sooner than the inside (neutral layer), thus reducing the apparent $E^{\prime} / y$ earlier than expected from average MC. The trends in $E^{\prime} / y$ in Figure $5 \mathrm{~b}$ resembled a hysteresis loop (although with small amplitude), this shape would be consistent with an effect of vibrational properties of the outer layer changing ahead of average MC. Such an effect is theoretically proved (Nishino et al. 1992b), yet experimentally its impact on dynamic modulus is small even on much thicker $(15 \mathrm{~mm})$ specimens than the $2 \mathrm{~mm}$ studied here (Nishino et al. 1992a). Hunt (1997), using vibrational tests on very thin $(0.5 \mathrm{~mm})$ specimens, states that destabilisation on resonance frequency, and hence on $E^{\prime} / \gamma$, are noticeable, but of such low amplitude that there are "unlikely to have an important influence on the material behaviour". On the other hand, Salmén and Olsson (2016) found that transient effects on dynamic modulus of elasticity are much more pronounced for very thin papers, than for thicker ones, and such an effect would not be related to moisture gradients, but to the speed of sorption.

On stabilised, "air-dry" (usually $20^{\circ} \mathrm{C}$ and $60-65 \% \mathrm{RH}$ ) wood, a very strong relation exists between $\tan \delta$ and $E^{\prime} / \gamma$ (Figure 6). This is evidenced statistically on thousands of specimens (Brémaud et al. 2012; Ono and Norimoto 1983, 1984,1985 ) and can be reconstructed theoretically by taking into account the microfibril and/or grain angles (Brémaud et al. 2011; Obataya et al. 2000). Schematically, vertical shifts from this standard curve reflect modifications of the amorphous matrix. In present results, spruce and maple in $\mathrm{L}$ direction, stabilised at $20^{\circ} \mathrm{C}$ and $65 \% \mathrm{RH}$, were slightly above the "standard" curve, as is usually observed for these two species (Brémaud et al. 2009, 2012). In $\mathrm{L}$ direction (Figure 6a), the effect of EMC dependence, 
shown in bold thick lines, in the range 35-85\% RH (Brémaud and Gril 2020), traced an oblique trend departing from the curve for natural variability at $65 \% \mathrm{RH}$, but that could easily be taken into account. But the transient destabilisation in $\tan \delta$ resulted in strong deviations from either the "air-dry variability curves", or the "EMC dependence curves".

This has important practical consequences. As stated above, changes in $\tan \delta$ through time could not be inferred from changes in MC or other properties and stable $\tan \delta$ could not be inferred from stable EMC. Figure 6 shows that transients in $\tan \delta$ could neither be inferred from the $\tan \delta-E^{\prime} / y$

a
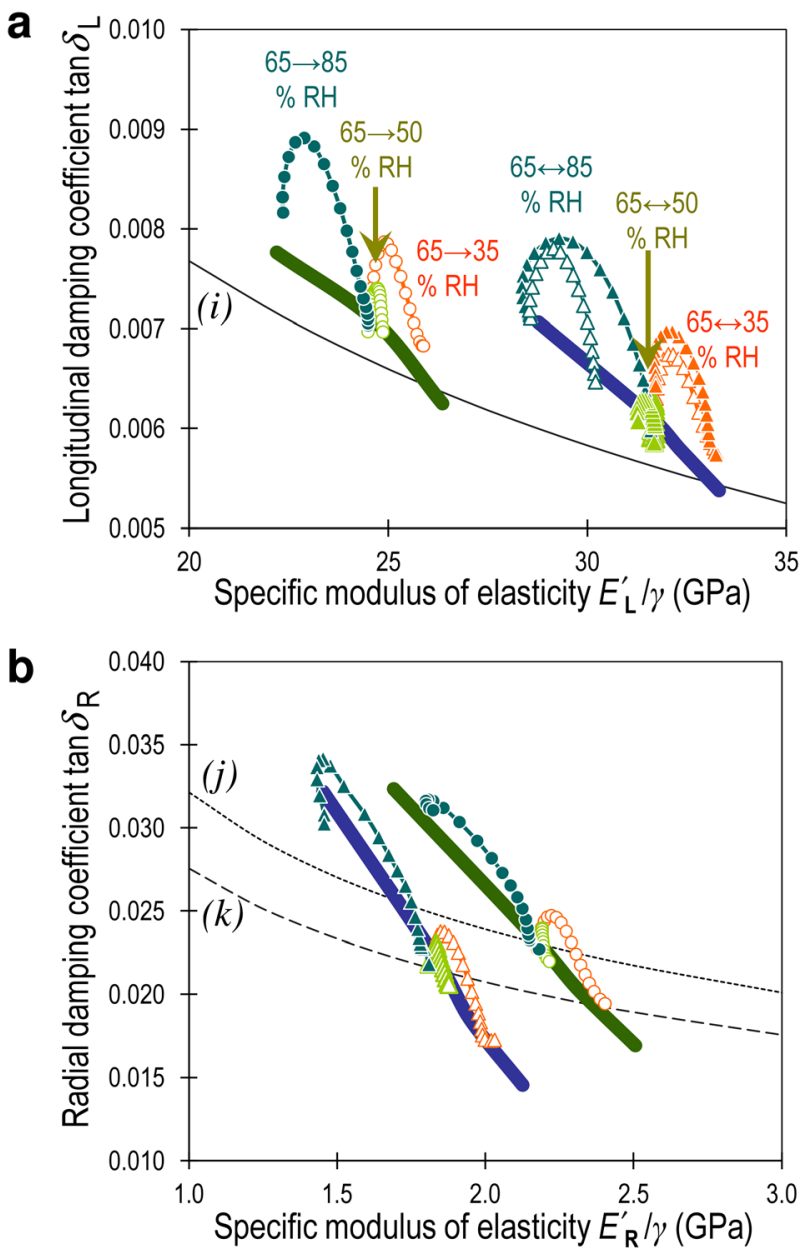

Figure 6: Relation between $\tan \delta$ and $E^{\prime} / \gamma$, in (a) $L$ and (b) R direction. Thick bold lines: EMC dependence in the range $35-85 \% \mathrm{RH}$ (Brémaud and Gril 2020). Symbols: transient values after stepchange in RH (starting from 65\% RH). Filled symbols: ad-sorption, open symbols: de-sorption. Triangles: spruce, circles: maple. Thin black curves: relations for variability of wood stabilized at "standard air-dry" condition, (i) softwoods and hardwoods in L direction (Brémaud et al. 2012; Ono and Norimoto 1983), (j): hardwoods in the three principal directions of anisotropy (Ono and Norimoto 1985), ( $k$ ) spruce with angles in the L-R plane (Ono and Norimoto 1983). diagram. EMC dependence of $\tan \delta$ is not sufficient to predict the behaviour in a new environment because the damping changes on a relatively short-time scale will always be above that expected from EMC, and above that detectable from other vibro-mechanical properties. The standard $\tan \delta-E^{\prime} / y$ relation is no longer valid under non-equilibrium conditions.

In $\mathrm{R}$ direction (Figure $6 \mathrm{~b}$ ), studied spruce and maple at "equilibrium air-dry" conditions matched with the curves for softwoods and hardwoods, respectively. The EMC dependence appeared prominent, before the natural variability. The destabilisation process, although clearly visible and in a comparable order of relative amplitude as in $\mathrm{L}$ direction (Figure 6a), appeared more in line with the EMC dependence.

\subsection{Destabilisation expressed by non-linear evolution of damping against moisture content}

In Figure 7, changes in $\tan \delta$ through time after step-change in conditions were plotted against corresponding changes in internal MC. This type of representation is used by Sasaki et al. (1988) and by Hunt and Gril (1996), but with very wide steps of RH changes, and by Hunt (1997) with successive small steps of RH. This representation highlights the differential effects of MC itself, and of transient destabilisation. Absolutely all conducted experiments showed a clear transient destabilisation, whatever the trajectory of conditions, the species, and the material direction. Trajectories including oven-dry or water-saturated state also exhibited a destabilisation effect, however they will not be further analysed because they involve additional phenomena. The destabilisation is thought to be an acceleration of normal time-dependent behaviour (Hunt and Gril 1996, Ishimaru et al. 2001), and is materialised by $\tan \delta$ always increasing then decreasing. A general shape of curves with increase then decrease along moistening (with reverse order in drying, unlike for $\tan \delta$ ) is observed for dimensional swelling and shrinkage where the explanation could involve internal stresses (Hunt 1997). The transient curves of $\tan \delta$ through time, showed some similarities with that for internal stresses developing as a result of moisture gradients inside specimens (Konopka and Kaliske 2018). Yet, the amplitude of curves of destabilisation in $\tan \delta$ plotted against MC obtained by (Hunt and Gril 1996) and by (Hunt 1997) on specimens of very small cross-section $\left(0.5 \times 3.2 \mathrm{~mm}^{2}\right)$ is at least equal, and rather larger, than the amplitude here observed on moderately small crosssection $\left(2 \times 12 \mathrm{~mm}^{2}\right)$, which suggests that gradients 


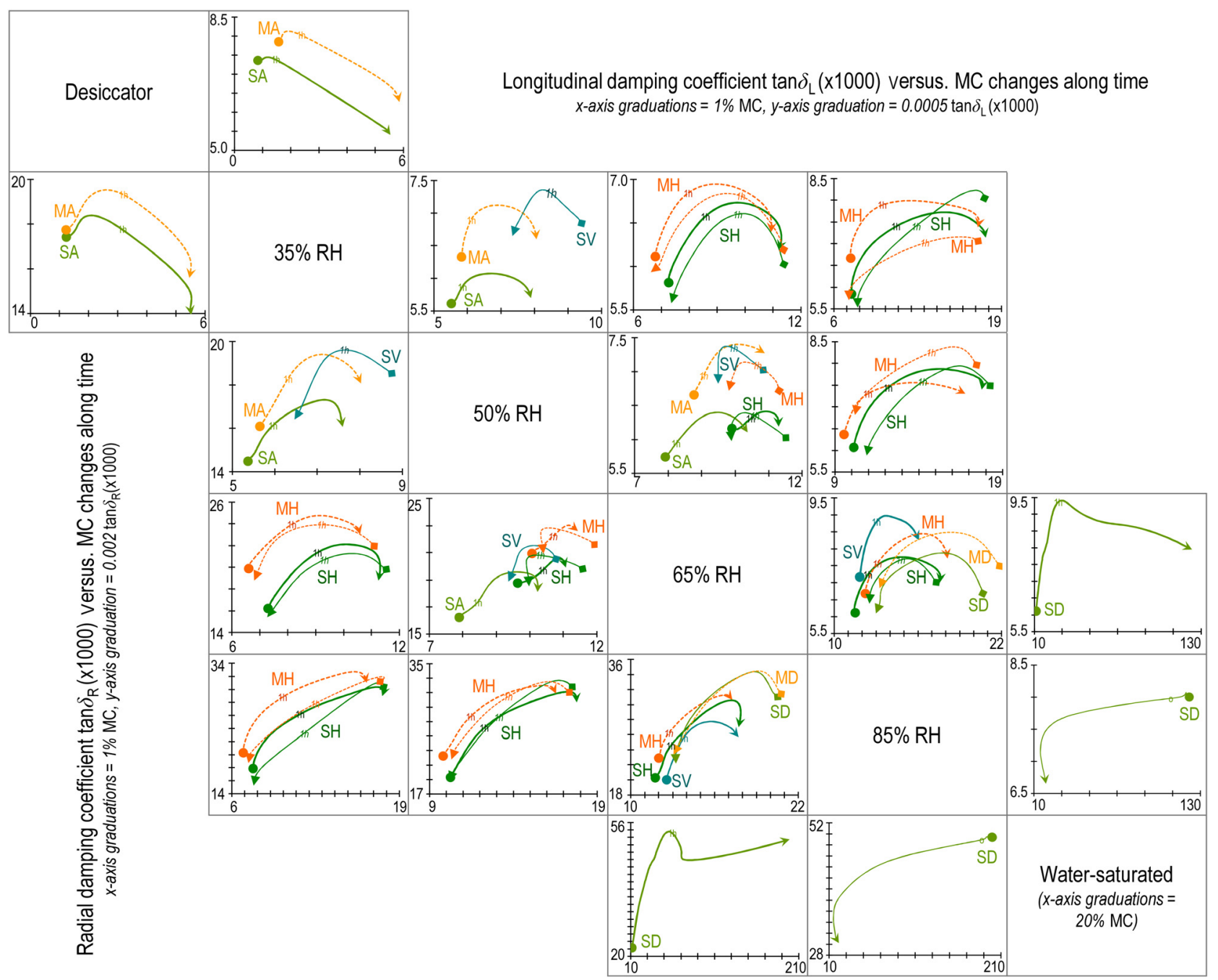

Figure 7: Matrix of changes in tan $\delta$ along time after step-change in conditions, plotted against concomitant changes in MC. Individual graphics correspond to a given pair of conditions (diagonal cells). Above diagonal: $L$ direction, below diagonal: $R$ direction. Plain green lines: spruce, dotted or dashed orange lines: maple; thick lines: adsorption, thin lines: desorption (arrows = direction of sorption). Different samplings and protocols are indicated by letters (S: spruce; M: maple; H, A, D, V: trajectories in Figure 1) and by different colour hues.

a

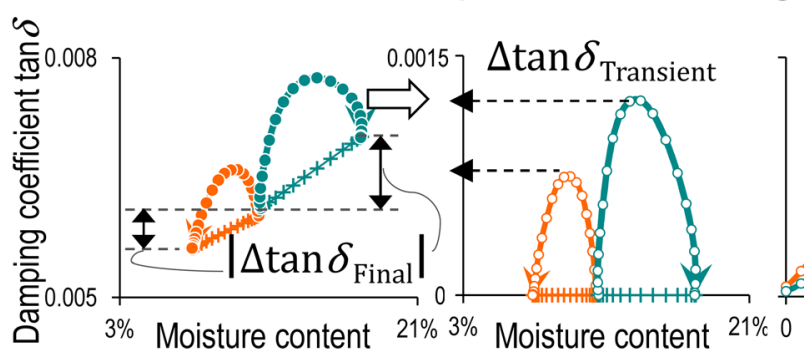

C

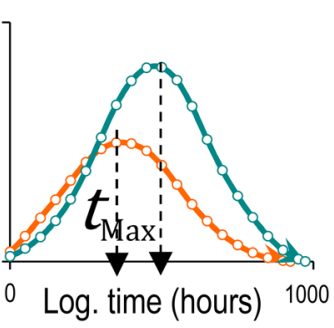

Figure 8: Quantification of respective effects of destabilisation, and of MC dependence, on $\tan \delta$. (a) Global changes in $\tan \delta$ and MC: determination of absolute increments $\left(\left|\Delta \tan \delta_{\text {Final }}\right|\right)$, and drawing a linear interpolation between initial and final values of $\tan \delta$ and MC. (b) Subtraction of the linear interpolation from the global curve of change in $\tan \delta$, to quantify the transient destabilisation $\Delta \tan \delta_{\text {Transient }}$ and its peak value. (c) Plotted against log time to quantify occurrence $\left(t_{\text {Max }}\right)$ of maximum destabilisation of $\tan \delta$.

effects, if any, are hidden by the molecular destabilisation. Also, although information on the effect of macroscopic stresses on wood material (not structural) damping is very limited, it does not plead for a significant effect (Geisler 
et al. 1994). An effect of internal stresses at a macroscopic scale is anyhow unlikely because, in the conditions of changing $\mathrm{RH}$ without external mechanical loading, the behaviour should remain linear, i.e. the material properties should not be affected by stress levels. However microstresses are likely at the molecular scale, i.e. within the conformation of cell-wall polymers (Hunt 1997, Obataya et al. 2020). In present results, for $\mathrm{RH}$ trajectories that involved a single protocol/sampling, the drying and the humidifying paths showed very similar trends, with very little hysteresis in destabilisation. This does not seem to support a hypothesis of structural effects where, as introduced in previous section, apparent flexural vibrational properties would change ahead of average MC due to gradients inside specimens and would result in a loop of hysteresis. Very little hysteresis also slightly contradicts hypotheses that drying could have a larger effect on destabilisation than humidifying (Hunt and Gril 1996); yet for mechanosorptive creep a single mechanism is suggested for drying or humidifying (Montero et al. 2012). Here, an experiment between 65 and $85 \%$ RH in L direction did show a larger effect of drying, but it belonged to the $\mathrm{D}$ (starting from water-saturated) protocol (the same drying path from $\mathrm{H}$ protocol did not show a larger destabilisation than in humidifying). In Figure 7, the individual RH steps that showed more dispersion all included several protocols (H, A, D or V, see Figure 1), i.e. dispersion was rather linked to the history and/or the natural variability of specimens. In general, the destabilisation curves appeared much more pronounced in the $\mathrm{L}$, than $\mathrm{R}$, direction, but it should be kept in mind that EMC dependence is much stronger in $\mathrm{R}$ direction.

\subsection{Quantifying the amplitude of destabilisation in damping}

As transient destabilisation in $\tan \delta$, and its MC dependence, happen concurrently, they should be separated in order to quantify their respective impact. Based on the representation in Figure 7, a quantification method was proposed (Figure 8). First, transient changes in $\tan \delta$ were plotted against those in MC (Figure 8a). The differences between final and initial values (considered at quasiequilibrium) were recorded as the increment in $\tan \delta$, noted $\left|\Delta \tan \delta_{\text {Final }}\right|$ (expressed in absolute values for comparing the ad- and de-sorption paths). A linear interpolation was made between initial and final values of $\tan \delta$ and of MC (i.e. assuming there was no destabilisation effect). Although, on the full hygroscopic range the EMC dependence of $\tan \delta$ is non-linear, a linear interpolation was an acceptable simplification in the range of 35-85\% RH on the material studied here (Brémaud and Gril 2020). This linear interpolation was subtracted from observed changes in $\tan \delta$ through time. The resulting curve (Figure $8 \mathrm{~b}$ ) allowed to quantify the absolute value of change in $\tan \delta$ due to destabilisation in itself, noted $\Delta \tan \delta_{\text {Transient }}$. When plotting the obtained curve against time since step-change in $\mathrm{RH}$ conditions, the time of occurrence of maximum destabilisation ( $t_{\text {Max }}$ in Figure 8c) could be determined.

This quantification was done on the 65 conducted experiments (Figure 9). The peak value of destabilisation in $\tan \delta$ increased with wider steps of EMC (except for the single widest step in drying). The trends of amplitude of destabilisation appeared to be rather symmetrical between drying and humidifying. In L direction (Figure 9a), the changes in $\tan \delta$ due to the destabilisation ( $\left.\Delta \tan \delta_{\text {Transient }}\right)$ were at least of the same order of magnitude as the changes due to EMC dependence (final increments $\left|\Delta \tan \delta_{\text {Final }}\right|$ ). For moderate humidity steps, the effect of destabilisation even exceeded that of EMC dependence. The amplitude of the destabilisation amounted to $0.0003-0.0015$ in $\tan \delta_{\mathrm{L}}$. Such values might seem "small", but, to give an idea of their order of magnitude, $0.0005-0.001$ differences in $\tan \delta_{\mathrm{L}}$ are sufficient to discriminate between average values per species (Brémaud et al. 2009, Brémaud 2012) - although within-species variability often exceeds the betweenspecies diversity. Within-species, differences in $\tan \delta_{\mathrm{L}}$ of 0.0004 have been found sufficient for expert musical instrument makers to discriminate between "excellent" and "good" quality wood (Hase 1987).

In $\mathrm{R}$ direction (Figure $9 \mathrm{~b}$ ), the destabilisation amounted to $0.0007-0.006$ changes in $\tan \delta_{\mathrm{R}}$. The pattern of evolution along EMC increments was similar to that in $\mathrm{L}$ direction. The destabilisation $\left(\Delta \tan \delta_{\text {Transient }}\right)$ was of the same order of magnitude as the EMC dependence $\left(\mid \Delta \tan \delta_{\text {Final }}\right)$ for small humidity steps $( \pm 5 \% \Delta \mathrm{EMC})$, however not superior as observed above in $\mathrm{L}$ direction. For wider steps in EMC the amplitude of destabilisation appeared significantly lower than the EMC-induced changes in $\tan \delta_{\mathrm{R}}$.

In order to better evaluate the relative significance of the changes in $\tan \delta$ induced by destabilisation, and by EMC dependence, in both $\mathrm{L}$ and $\mathrm{R}$ directions, these changes $\left(\left|\Delta \tan \delta_{\text {Final }}\right|\right.$ and $\left.\Delta \tan \delta_{\text {Transient }}\right)$ were normalised through dividing them by the average value per species and directions at quasi-equilibrium in "standard air-dry" conditions $\left(20^{\circ} \mathrm{C}\right.$ and $65 \% \mathrm{RH}$, resulting in EMC of $11.6 \%$ for spruce and $11.9 \%$ for maple). These reference values were 0.0058 (spruce) and 0.0071 (maple) for $\tan \delta_{\mathrm{L}}$ and 0.0204 (spruce) and 0.0222 (maple) for $\tan \delta_{\mathrm{R}}$ (Brémaud and Gril 


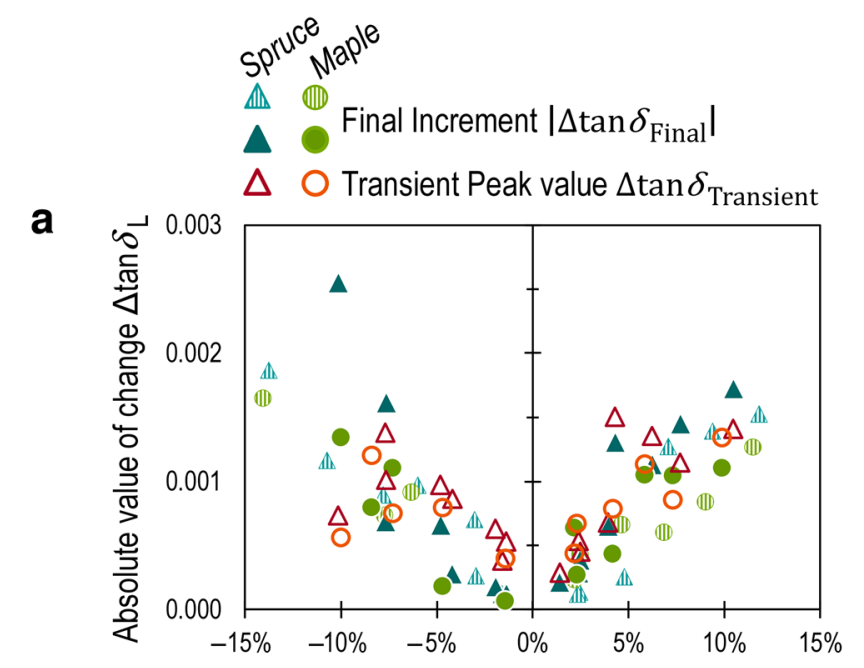

b
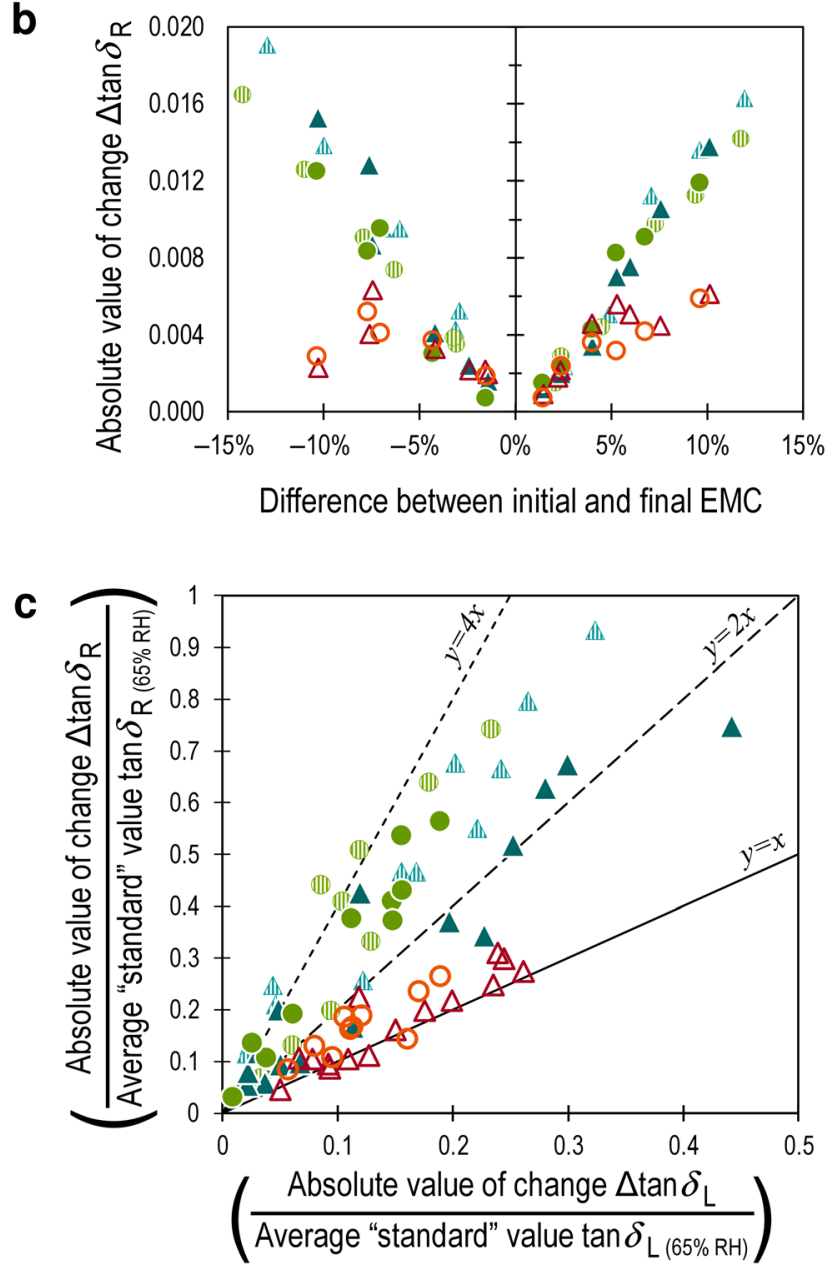

Figure 9: Quantified amplitudes of changes in tan $\delta$ due to transient destabilisation (open symbols) and to MC dependence (filled symbols), (dashed symbols: RH steps measured at final stage but not during transients). Triangles: spruce, circles: maple. (a) Longitudinal ( $\mathrm{L}$ ) and (b) radial (R) $\tan \delta$. (c) Comparison of changes in R and L directions: amplitude of changes were normalised by average values (per species and direction) at quasi-equilibrium in "standard air-dry" conditions of $20{ }^{\circ} \mathrm{C}$ and $65 \%$ RH (Brémaud and Gril 2020).
2020). Based on normalised values, maple had a bit smaller $\Delta \tan \delta_{\text {Transient }}$ and clearly smaller $\left|\Delta \tan \delta_{\text {Final }}\right|$ than spruce in $\mathrm{L}$ direction, but only slightly so in $\mathrm{R}$ direction. In this representation (Figure 9c), the changes in damping due to transient destabilisation amounted to up to $30 \%$ of the average standard values of $\tan \delta$, both for $\mathrm{L}$ and $\mathrm{R}$ directions. The effect of destabilisation was comparable, having in average only $+25 \%$ higher relative amplitude in $\mathrm{R}$, than in $\mathrm{L}$ direction. Yet, the relative influence of EMC dependence was much (2-4 times) higher in $\mathrm{R}$ (amounting to up to $100 \%$ of reference values of $\tan \delta_{\mathrm{R}}$ ) than in L direction (maximum 45\%, and usually less than $30 \%$ of reference values of $\tan \delta_{R}$ ). The very strong EMC dependence of $\tan \delta$ in $\mathrm{R}$ direction overshadowed the amplitude of the destabilisation effect (Figure 9c), thereby explaining some unexpected trends in some raw experimental figures (apparently delayed $\tan \delta$ peak in R in Figure 4, less pronounced $R$ curves in Figure $6 \mathrm{~b}$ and Figure 7 ), while in fact this destabilisation effect was of the same order in $\mathrm{R}$ and $\mathrm{L}$ direction. Nevertheless, vibrational properties of wood in $\mathrm{R}$ direction should be mostly dictated by the amorphous, quasi isotropic matrix (Norimoto et al. 1986). If the destabilisation depended only on matrix behaviour, then the ratio between $\mathrm{R}$ and $\mathrm{L}$ direction should be the same for $\Delta \tan \delta_{\text {Transient }}$ as for $\left|\Delta \tan \delta_{\text {Final }}\right|$. Thus, the comparable relative amplitude of $\Delta \tan \delta_{\text {Transient }}$ between $\mathrm{L}$ and $\mathrm{R}$ suggests that, at the cell-wall scale, the destabilisation phenomenon would somehow involve the anisotropic structure of the microfibrillar network.

\subsection{Occurrence of maximum destabilisation in damping}

To explore kinetic aspects of the transient changes in damping along with changes in moisture, their 1st derivative against log time were studied (Figure 10a). That for mass of specimen, $\mathrm{d} m / \mathrm{d} \log t$, was named "sorption rate" and compared to the destabilisation in damping $\left(\Delta \tan \delta_{\text {Transient }}\right)$ in Figure 10b, c.

Within a given experiment, the time $\left(t_{\text {Max }}\right)$ when the maximum amplitude of $\Delta \tan \delta_{\text {Transient }}$ occurred was nearly concomitant with that of maximum sorption rate (Figure 10b, c). Between different experiments, the maximum sorption rate was a correct indicator of the amplitude of the maximum $\Delta \tan \delta_{\text {Transient }}$ (Figure 10d). As expected, this maximum sorption rate was linearly and very strongly correlated to steps in EMC, following a single trend for ad- and de-sorption, for spruce and maple, and for $\mathrm{R}$ and $\mathrm{L}$ directions. This explains the high similarity in trends between Figure 10d and Figure 9a, b. 

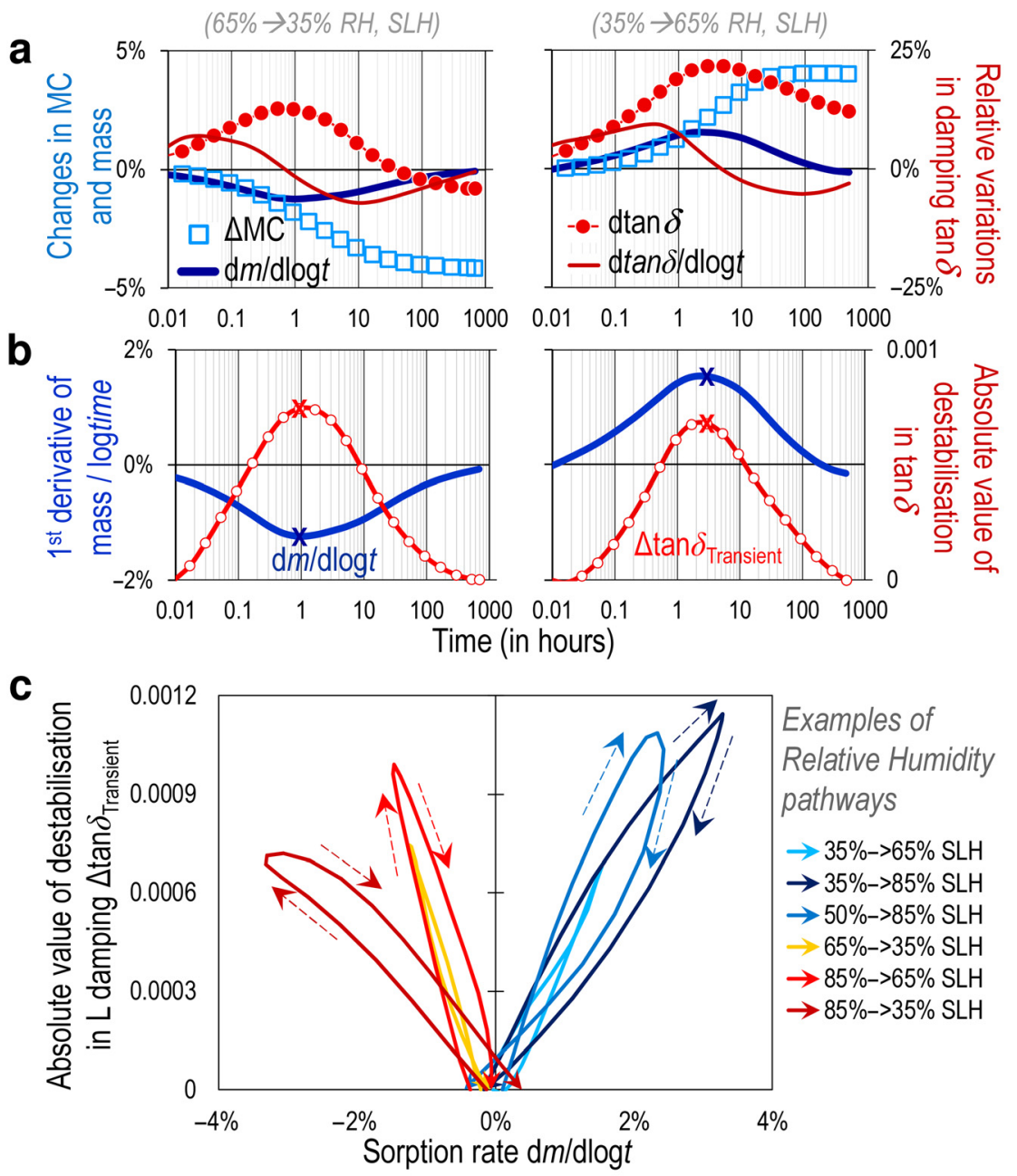
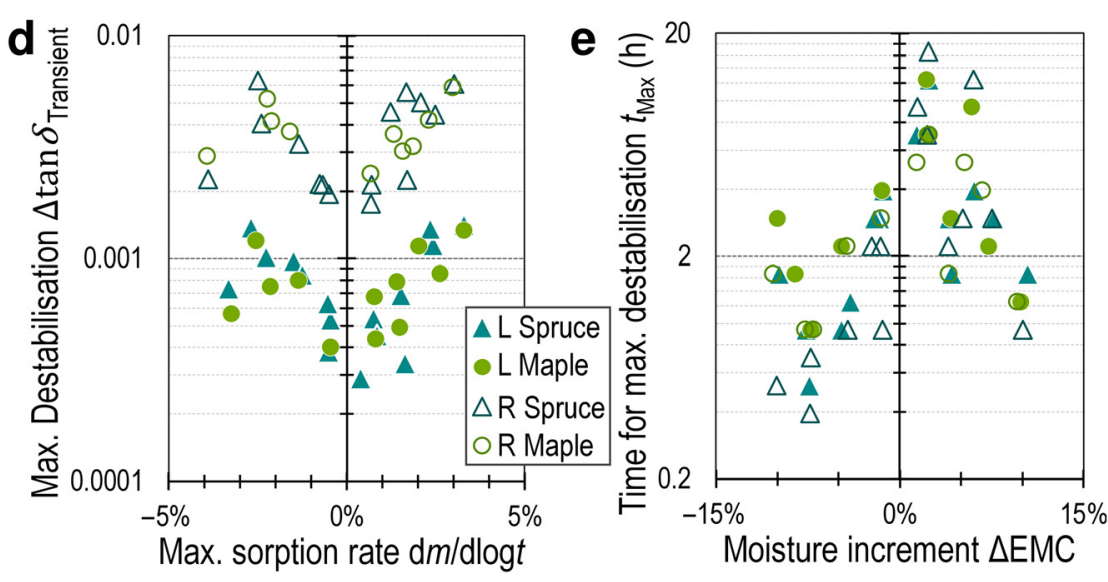

Figure 10: Time-quantification of maximum destabilisation. (a) Changes in MC, mass and $\tan \delta$ and their 1st derivative against log time. (b) Time evolution of 1st derivative of mass changes (sorption rate) and of $\Delta \tan \delta_{\text {Transient }}, \mathrm{X}=$ identified maxima. (c) Evolution of $\Delta \tan \delta_{\text {Transient }}$ plotted against that of sorption rate, illustrated on a few RH steps on longitudinal spruce. (d, e) Time parameters quantified on all conducted experiments (filled symbols: L, open symbols: $R$ direction, triangles: spruce, circles: maple). (d) Maximum amplitude of destabilisation as a function of maximum sorption rate; (e) Time for occurrence of maximum destabilisation $\left(t_{\text {Max }}\right)$ as a function of EMC increment.
The influence of sorption speed on the amplitude of transient effects is evidenced for various polymeric fibres such as wool (Back et al. 1983; Wang et al. 1991), in dynamic mechanical analysis of paper (Salmén and Olsson 2016), and in wood mechano-sorptive creep (Takahashi et al. 2006). Mechanisms underlying transient moisture effects on semi-amorphous polymeric materials are classified as hydrogen bonding, slip-planes and crystallite slippage (Wang et al. 1991). Moisture sorption may not proceed homogeneously, but instead take place in priority at the interface between amorphous hemicelluloses and crystalline cellulose microfibrils (Kulasinski et al. 2015, 2017). A delay is found between the crystalline arrangement, the heat flow of sorption, and the MC changes; i.e. 
rearrangement of fine structure of wood would not strictly follow moisture sorption (Sugino et al. 2007). In present results, the concomitance of maximum destabilisation with maximum sorption rate (Figure $10 \mathrm{~b}, \mathrm{c}$ ), and its anisotropic relative impact (i.e. similar in $L$, but lower in $R$ than would be expected from EMC dependence, Figure 9c), seemed to support the hypothetical mechanism of slippage at interfaces between microfibrils and matrix, in addition to the hydrogen bond breaking process (Hunt and Balsan 1996; Wang et al. 1991).

Between different experiments, however, it was not simple to predict the $t_{\text {Max }}$. The maximum sorption rate occurred much sooner (20-100 min) in desorption, and specially sooner for small EMC steps, than in adsorption $(1.5-50 \mathrm{~h})$, where smaller EMC steps reached this maximum rate later. The maximum sorption rate occurred about $\times 1.5$ earlier in $\mathrm{R}$ than $\mathrm{L}$ specimens, and about $\times 1.8$ later in maple than spruce, despite all reaching similar maximum values (determined by each EMC steps). The time ( $\left.t_{\text {Max }}\right)$ of occurrence of maximum $\Delta \tan \delta_{\text {Transient }}$ followed a comparable pattern but less contrasted (Figure 10e), because in adsorption the evolution of $\Delta \tan \delta_{\text {Transient }}$ tended to precede the maximum sorption rate, while in desorption it was either concomitant or slightly delayed (Figure 10c). The latter, however, was mostly observed for wide steps in EMC, while for smaller steps the hysteresis loop between these two parameters was very little (Figure 10c). This hysteresis over wider EMC steps might express second-order effects of moisture gradients inside the specimens, i.e. of vibrational properties to change ahead of average MC. The maximum $\Delta \tan \delta_{\text {Transient }}$ occurred after $5 \mathrm{~h}$ in average (20 min - 17 h) in adsorption (equivalent times for spruce and maple), and after $1.7 \mathrm{~h}$ in average ( $20 \mathrm{~min}-5 \mathrm{~h}$ ) in desorption (where it tended to be delayed by approx. $\times 1.8$ in maple). It tended to occur sooner $(\times 1.15$ when moistening, $\times 1.5$ when drying) in $\mathrm{R}$ than $\mathrm{L}$ direction.

Some parts of present results are in line with Hunt and Gril (1996): $\tan \delta$ changing faster than MC in initial stages of humidifying, more slowly than MC in initial stages of drying. But globally present results contradict the hypothesis that " $\tan \delta$ changes would be much slower in drying" (Hunt and Gril 1996). This hypothesis was however based on very wide steps of RH (35-81-42\%) without subtracting EMC dependence from the transient changes. Over small humidity steps (EMC increments $\pm 2.5 \%$ ), Hunt (1997) calculated that characteristic times are nearly 3 times (for moisture) or 5 times (for "primary change in $\tan \delta$ ", i.e. destabilisation) faster in drying than in humidifying. This goes in the same direction as present results on destabilisation, and will be further discussed in next section for the following process of change in $\tan \delta$, related to physical ageing.

\subsection{Estimating the approach to "equilibrium" in a physical ageing process}

From a fundamental point of view of wood physics, little is known about the slow reach to equilibrium after destabilisation, i.e. about physical ageing, in the domain of dynamic, vibrational properties, exception made from evidence in the few previously cited articles (Gril 1988; Hunt 1997; Hunt and Balsan 1996; Hunt and Gril 1996; Obataya et al. 2020; Suzuki 1962; Sasaki et al. 1988).

From a practical point of view, many experimental works consider that measuring "equilibrium" physicalmechanical properties can be done as soon as MC appears stable, i.e. when further changes are sufficiently small for not being experimentally detectable. Present results showed that this could not be a criterion as far as viscoelastic damping is considered. Classically, experimental characterisations of vibrational properties are considered "at equilibrium" after 2-3 weeks in controlled conditions, but applied stabilisation times do vary between publications (Brémaud and Gril 2020). Even if it is known that reaching "equilibrium" is a slow, asymptotic phenomenon (Gril 1988; Hunt and Gril 1996; Ishimaru et al. 2001), would there be a means to evaluate if an "equilibrium" in a given property is reached or on the way to be reached?

A method to answer this question was proposed in Figure 11. The evolution through time of measured values for a given property (mass of specimens, to monitor MC, in Figure 11a, and $\tan \delta_{\mathrm{L}}$ in Figure 11b) was plotted against the 1st derivative of relative variations in this property with $\log$ time (i.e. its "rate of change"). When the rate of change ( $x$ axis) tended towards zero, the corresponding value ( $y$-axis) of property could be considered close to equilibrium. Even if the rate of change was not yet zero, the trend of the curve might be extended to estimate the future "equilibrium" value (on $y$-axis), in cases where curves were clearly returning towards zero of $x$-axis.

The application of this method to all studied $\mathrm{RH}$ steps is presented in Figure 11, for spruce in L direction. For MC, analysed through the direct measurements of changes in mass of the specimens (Figure 11a), virtually all conducted experiments had reached equilibrium, even those with relatively short time of monitoring (813 days). Only three experiments had not fully completed the loop returning to null change rate (although they 
a

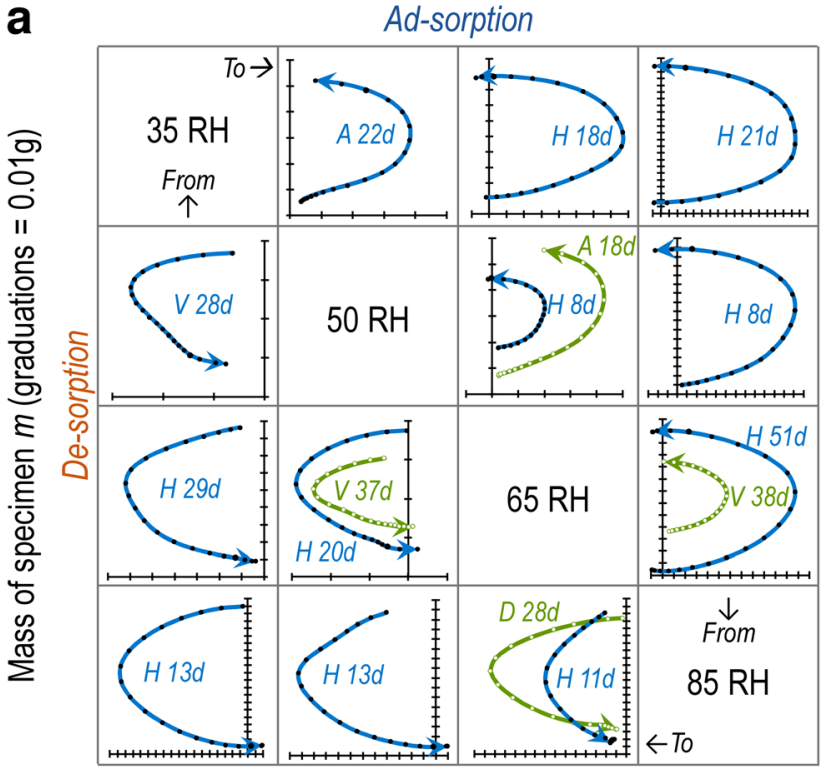

1 st derivative of mass change versus. time: $\mathrm{d} m / \mathrm{dlog} t \quad$ (graduations $=0.2 \%$ )

b

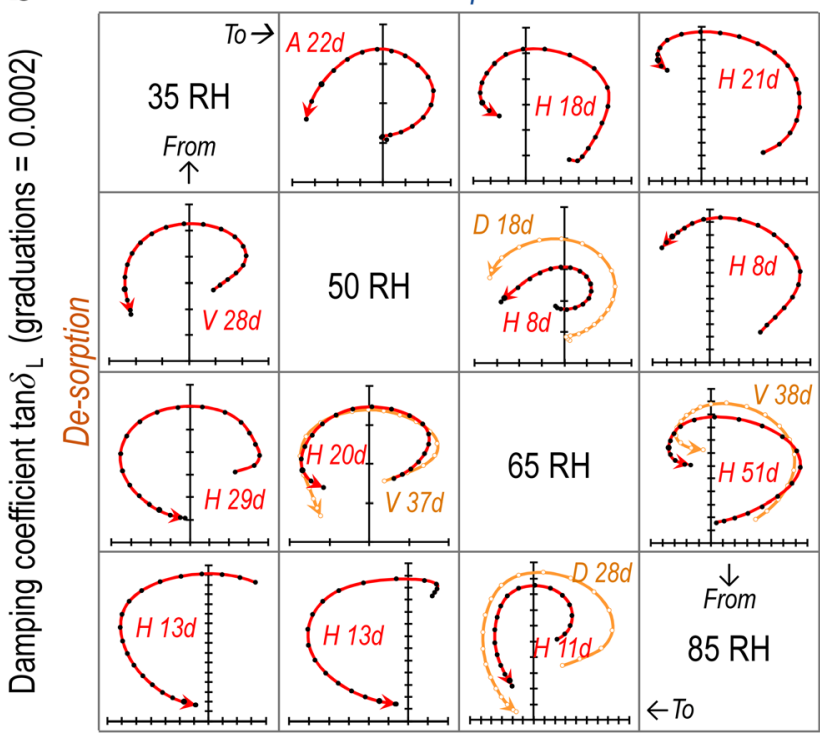

1st derivative of damping changes versus. time: $\mathrm{d} \tan \delta_{L} / \mathrm{dlog} t$ (graduations $\left.=2 \%\right)$

Figure 11: Evaluating if equilibrium values are approached, for (a) MC (expressed by mass of specimens) and (b) $\tan \delta . y$-axis: values measured through time; $x$-axis: 1 st derivative of variations through time (=rate of change). Amplitude of changes can be read from the number of (constant) graduations. Diagonal cells: Initial and final $\mathrm{RH}$, above-diagonal: humidifying, below-diagonal: drying. The protocol (see Figure 1) and number of days of each experiment is indicated. Spruce in L direction.

were conducted on 18-28 days). These three experiments corresponded to small MC steps, in the low-RH domain, i.e. to slow sorption rate.
Results on $\tan \delta$ (Figure 11b) were very contrasted with those on MC: only five experiments had reached (or nearly reached) equilibrium, and these included some of the longest durations of monitoring (29-51 days). This is consistent with the statement of much slower reaching to equilibrium for dynamic viscoelastic properties, than for $\mathrm{MC}$, i.e. with the concept of physical ageing on these properties (Hunt and Gril 1996, Hunt 1997). However, some experiments monitored on relatively short time (13 days for example from 85 to $50 \%$ and from 85 to $35 \% \mathrm{RH}$ ) had reached equilibrium, while some experiments monitored over longer times (22-28 days for example between 50 and $35 \% \mathrm{RH}$ ) were still far from a forecast of equilibrium. Due to practical constraints, not all experiments could be monitored along a similarly long duration, and this is a limitation of the present study because a general predictive model of equilibration time could not yet be proposed. However, Figure 11b allowed to draw some preliminary conclusions. Most experiments in desorption exhibited at least a beginning of return path towards null rate of change in $\tan \delta$, while most of the experiments in adsorption did not show this return path, or solely a beginning of inflexion in the curve, regardless of duration of monitoring. This seemed consistent with results of Hunt (1997), where "secondary changes" in $\tan \delta$ have characteristic times 4 times higher in ad- than in de-sorption. In Figure 11b, most of the experiments over moderate RH steps (i.e. close to the diagonal) appeared still quite far from reaching equilibrium, while experiments over wide RH and EMC steps (i.e. far from the diagonal) appeared closer to reach equilibrium, regardless of their duration of monitoring. This suggested that wider humidity steps, with faster sorption, could bring about a larger amplitude of destabilisation (Figure 9a, b, Figure 10d), but that appeared sooner (Figure 10e), and that sooner recovered towards an "equilibrium" value, than smaller humidity steps which would induce smaller destabilisation (however often larger than EMC dependence, Figure 9a), but lasting over a longer time.

\section{Conclusion}

The goal of this article was to explore the transient phases of vibrational properties of wood when changing humidity. A wide campaign (65 experiments lasting 1-7 weeks) of monitoring changes in properties through time after stepchanges in conditions, combined with data analysis methods to separate and quantify the intertwined phenomena (EMC dependence, destabilisation, and physical ageing), indicated that: 
- Damping $(\tan \delta)$ always increased rapidly after changing humidity, whether the moisture increased or decreased. EMC could be reached within one week, while $\tan \delta$ might not reach equilibrium before several weeks. Thus, neither MC nor reaching EMC were indicators of $\tan \delta$.

- Compared to $\tan \delta$, specific modulus of elasticity $\left(E^{\prime} / \gamma\right)$ was less affected by EMC dependence, and much less subject to transient effects. Thus, $E^{\prime} / y$ neither could be an indicator of stabilised $\tan \delta$. And the wellestablished standard $\tan \delta-E^{\prime} / \gamma$ relation was no longer valid under non-equilibrium conditions.

- For moderate steps in RH/EMC, the transient destabilisation in $\tan \delta$ equalled, or even exceeded (in $\mathrm{L}$ direction) the EMC dependence.

- The amplitude of destabilisation in $\tan \delta$ was determined by the width of humidity steps and the sorption rate (along logarithm of time), but not by the direction of sorption (drying or humidifying).

- The time for occurrence of maximum destabilisation in $\tan \delta$ was nearly concomitant with maximum sorption rate within a given test (but not between different $\mathrm{RH}$ steps).

- The kinetics of transient processes in $\tan \delta$ was influenced by the direction of sorption (maximum destabilisation occurred sooner, but was also recovered sooner, in drying than in humidifying) and by the width of RH/EMC steps (smaller increments in moisture caused smaller destabilisation, but that occurred later, and lasted over longer time before to recover equilibrium).

- The phenomena were similar for spruce and maple, although maple (denser and less anisotropic) had a bit slower kinetics and smaller destabilisation in L (less so in $\mathrm{R})$.

- Transient destabilisation on $\tan \delta$ in $\mathrm{R}$ direction had higher absolute values, but similar relative amplitude compared to L direction, while EMC dependence was much stronger in R. It suggested that the cell-wall origin may not be only in the quasi-isotropic matrix.

Obtained quantitative results confirm the hypothesis previously put forward by one of the authors, that "destabilisation followed by physical ageing might explain various anomalies observed in wood behaviour" (Hunt and Gril 1996). Such transient behaviours should be carefully examined and taken into account when designing experimental research protocols, as they could probably modify either the fine quantitative values, or sometimes even some elements of conclusions, of many previous works about vibrational (and more generally dynamic mechanical) properties, including some by the authors. Practical consequences in real-life uses are also important, in the case of wooden musical instruments for instance, but also in all other cases where either energy dissipation by internal friction, or vibration/noise control, might be involved.

Acknowledgments: Authors are grateful to the generous help of Tancrède Alméras, CNRS Researcher at LMGC Montpellier, who developed the smoothing and interpolation algorithm used for post-treatment of experimental data. The research benefited from funding of CNRS and of Région Languedoc-Roussillon, and from access to the experimental facilities of BioWooEB Lab in CIRAD, Montpellier, where the kind support of D. Guibal, J. Gérard and P. Langbour is acknowledged. Experiments were initiated by the 1st author at the fall of 2012 while ending a Post-Doc (Marie Curie Fellowship) at Wood Laboratory of Empa, Switzerland, where the kind support of F.W.M.R. Schwarze, R. Widmann and D. Heer is acknowledged. The authors wish to thank three anonymous reviewers for their very relevant comments that helped improving the final manuscript.

Author contribution: All the authors have accepted responsibility for the entire content of this submitted manuscript and approved submission.

Research funding: None declared.

Conflict of interest statement: The authors declare no conflicts of interest regarding this article

\section{References}

Armstrong, L.D. and Kingston, R.S.T. (1960). Effect of moisture changes on creep in wood. Nature 185: 862-863.

Back, E.L., Salmén, L., and Richardson, G. (1983). Transient effects of moisture sorption on the strength properties of paper and woodbased materials. Sven. Papperstidn. 6 - 1983 - 86: 61-71.

Borland, M.J. (2014). The effect of humidity and moisture content on the tone of musical instruments. PhD In: Systems design engineering. University of Waterloo, Canada, p. 161.

Brémaud, I. (2006). Diversity of woods used or usable in musical instruments making. PhD In: Mechanics, Vol. 2. University Montpellier, p. 302.

Brémaud, I. (2012). Acoustical properties of wood in string instruments soundboards and tuned idiophones: biological and cultural diversity. J. Acoust. Soc. Am. 131: 807-818.

Brémaud, I., El Kaïm, Y., Guibal, D., Minato, K., Thibaut, B., and Gril, J. (2012). Characterisation and categorisation of the diversity in viscoelastic vibrational properties between 98 wood types. Ann. For. Sci. 69: 373-386.

Brémaud, I., Gril, J., and Thibaut, B. (2011). Anisotropy of wood vibrational properties: dependence on grain angle and review of literature data. Wood Sci. Technol. 45: 735-754.

Brémaud, I., Minato, K., and Thibaut, B.(2009). Mechanical damping of wood as related to species classification: a preliminary survey In: 
6th Plant Biomechanics Conference PBM09. 16-21 November 2009, Cayenne, French Guyana. Kourou: UMR EcoFoG. pp. 536-542.

Brémaud, I., Ruelle, J., Thibaut, A., and Thibaut, B. (2013). Changes in viscoelastic vibrational properties between compression and normal wood: roles of microfibril angle and of lignin. Holzforschung 67: 75-85.

Brémaud, I. and Gril, J. (2020). Moisture content dependence of anisotropic vibrational properties of wood at quasi equilibrium: analytical review and multi-trajectories experiments. Holzforschung. https://doi.org/10.1515/hf-2020-0028.

Dlouha, J., Gril, J., Clair, B., and Alméras, T. (2009). Evidence and modelling of physical aging in green wood. Rheol. Acta. 48: 333-342.

Ebrahimzadeh, P.R. (1997). Dynamic mechanical studies of wood, paper and some polymers subjected to humidity changes. $\mathrm{PhD}$. Chalmers University of Technology, Chalmers. Tekniska Högskola - Institutionen för polymera material.

Ebrahimzadeh, P.R. and Kubät, D.G. (1993).Effects of humidity changes on damping and stress relaxation in wood. J. Mater. Sci. Lett. 28: 5668-5674.

Engelund, E.T. and Salmén, L. (2012).Tensile creep and recovery of Norway spruce influenced by temperature and moisture. Holzforschung 66: 959-965.

Fioravanti, M., Goli, G., and Carlson, B. (2013). Viscoelastic and mechano-sorptive studies applied to the conservation of historical violins: a case study of the Guarneri "del Gesù" violin (1743) known as the "Cannone". J. Cult. Herit. 14: 297-303.

Fukada, E. (1950) The vibrational properties of wood I. J. Phys. Soc. Jpn. 5: 321-327.

Geisler, T., Skalmierski, B., and Sochacki, W. (1994) Influence of initial stress and varnish coatings on vibration damping in wooden beams. J. Theor. Appl. Mech. 32: 439-446.

Goli, G., Fioravanti, M., Busoni, S., Carlson, B., and Mazzanti, P. (2012) Measurement and modelling of mass and dimensional variations of historic violins subjected to thermo-hygrometric variations: the case study of the Guarneri "del Gesū" violin (1743) known as the "Cannone". J. Cult. Herit. 13S: S154-S160.

Gril, J. (1988). Une modélisation du comportement hygro-rhéologique du bois à partir de sa microstructure. PhD In: Physics. Université Paris 6, p. 268.

Hase, N. (1987). A comparison between acoustic physical factors of Honduras rosewood for marimbas and xylophones and a sensory evaluation of these instruments. Mokuzai Gakkaishi. 33: 762-768.

Hunt, D.G. (1990). Longitudinal shrinkage-moisture relations in softwood. J. Mater. Sci. 25: 3671-3676.

Hunt, D.G. (1997). Dimensional changes and creep of spruce, and consequent model requirements. Wood Sci. Technol. 31: 3-16.

Hunt, D.G. and Balsan, E. (1996). Why old fiddles sound sweeter. Nature 379: 681.

Hunt, D.G. and Gril, J. (1996). Evidence of a physical ageing phenomenon in wood. J. Mater. Sci. Lett. 15: 80-82.

Ishimaru, Y., Oshima, K., and lida, I. (2001). Changes in the mechanical properties of wood during a period of moisture conditioning. J. Wood Sci. 47: 254-261.

Konopka, D. and Kaliske, M. (2018). Transient multi-fickian hygromechanical analysis of wood. Comput. Struct. 197: 12-27.

Kulasinski, K., Derome, D., and Carmeliet, J. (2017). Impact of hydration on the micromechanical properties of the polymer composite structure of wood investigated with atomistic simulations. J. Mech. Phys. Solid. 103: 21-235.
Kulasinski, K., Guyer, R., Derome, D., and Carmeliet, J. (2015). Water adsorption in wood microfibril-hemicellulose system: role of the crystalline-amorphous interface. Bio. Macromol. 16: 2972-2978.

Montero, C., Gril, J., Legeas, C., Hunt David, G., and Clair, B. (2012). Influence of hygromechanical history on the longitudinal mechanosorptive creep of wood. Holzforschung 66: 757-764.

Navi, P., Pittet, V., and Plummer, C.J.G. (2002). Transient moisture effects on wood creep. Wood Sci. Technol. 36: 447-462.

Nishino, Y., Nakao, T., Tanaka, C., and Takahashi, A. (1992a). Changes in apparent Young's moduli of wood obtained by flexural and longitudinal vibrations with moisture adsorbing and desorbing processes. Mokuzai Gakkaishi 38: 429-432.

Nishino, Y., Nakao, T., Tanaka, C., and Takahashi, A. (1992b). Free-free beam vibration of wood with moisture gradients I. Analyses of the apparent Young's modulus obtained by fundamental mode applying theoretical method. Mokuzai Gakkaishi 38: 825-829.

Norimoto, M., Tanaka, F., Ohogama, T., and Ikimune, R. (1986). Specific dynamic Young's modulus and internal friction of wood in the longitudinal direction (in Japanese). Wood Res. Tech. Notes: 53-65.

Obataya, E., Norimoto, M., and Gril, J. (1998). The effects of adsorbed water on dynamic mechanical properties of wood. Polym. 39: 3059-3064.

Obataya, E., Ono, T., and Norimoto, M. (2000). Vibrational properties of wood along the grain. J. Mater. Sci. 35: 2993-3001, 6317.

Obataya, E., Zeniya, N., and Endo-Ujiie, K. (2020). Effects of seasoning on the vibrational properties of wood for the soundboards of string instruments. J. Acoust. Soc. Am. 147: 998-1005.

Ono, T. and Kataoka, A. (1979). The frequency dependence of the dynamic Young's modulus and internal friction of wood used for the soundboard of musical instruments II. The dependence of the Young's modulus and internal friction on frequency, and the mechanical frequency dispersion (in Japanese). Mokuzai Gakkaishi 25: 535-542.

Ono, T. and Norimoto, M. (1983). Study on Young's modulus and internal friction of wood in relation to the evaluation of wood for musical instruments. Jpn. J. Appl. Phys. 22: 611-614.

Ono, T. and Norimoto, M. (1984). On physical criteria for the selection of wood for soundboards of musical instruments. Rheol. Acta. 23: 652-656.

Ono, T. and Norimoto, M. (1985). Anisotropy of dynamic Young's Modulus and internal friction in wood. Jpn. J. Appl. Phys. 24: 960-964.

Peng, H., Jiang, J., Lu, J., and Cao, J. (2019). Orthotropic mechanosorptive creep behavior of Chinese fir during the moisture adsorption process determined in tensile mode via dynamic mechanical analysis (DMA). Holzforschung 73: 229-239.

Ranta-Maunus, A. (1975). The viscoelasticity of wood at varying moisture content. Wood Sci. Technol. 9: 189-205.

Salmén, L. and Olsson, A.-M. (2016). Physical properties of cellulosic materials related to moisture changes. Wood Sci. Technol. 50: 81-89.

Sasaki, T., Norimoto, M., Yamada, T., and Rowell, R.M. (1988). Effect of moisture on the acoustical properties of wood (in Japanese). Mokuzai Gakkaishi 34: 794-803.

Struik, L.C.E. (1977). Physical aging in amorphous polymers and other materials. Doctoral Thesis In: Applied sciences. TU Delft, Netherlands, p. 249.

Sugino, H., Sugimoto, H., Miki, T., and Kanayama, K. (2007). Fine structure changes of wood during moisture adsorption and 
desorption process analyzed by X-ray difraction measurement (in Japanese). Mokuzai Gakkaishi 53: 82-89.

Suzuki, M. (1962). The effects of water-sorption and temperature on dynamic Young's modulus and logarithmic decrement of wood. Mokuzai Gakkaishi 8: 13-18.

Takahashi, C., Ishimaru, Y., lida, I., and Furuta, Y. (2004). The creep of wood destabilized by change in moisture content. Part 1: the creep behaviors of wood during and immediately after drying. Holzforschung 58: 261-267.

Takahashi, C., Ishimaru, Y., lida, I., and Furuta, Y. (2005). The creep of wood destabilized by change in moisture content. Part 2: the creep behaviors of wood during and immediately after adsorption. Holzforschung 59: 46-53.

Takahashi, C., Ishimaru, Y., lida, I., and Furuta, Y. (2006). The creep of wood destabilized by change in moisture content. Part 3: the influence of changing moisture history on creep behavior. Holzforschung 60: 299-303.
Torres, J.A., de Icaza-Herrera, M., and Castaño, V.M. (2014). Guitar acoustics quality: shift by humidity variations. Acta Acust. Unit. Acust. 100: 537-542.

Wang, J.Z., Dillard, D.A., and Kamke, F.A. (1991). A review. Transient moisture effects in materials. J. Mater. Sci. 26: 5113-5126.

Zhan, T., Jiang, J., Peng, H., and Lu, J. (2016). Dynamic viscoelastic properties of Chinese fir (Cunninghamia lanceolata) during moisture desorption processes. Holzforschung 70: 547-555.

Zhan, T., Jiang, J., Lu, J., Zhang, Y., and Chang, J. (2018a). Influence of hygrothermal condition on dynamic viscoelasticity of Chinese fir (Cunninghamia lanceolata). Part 2: moisture desorption. Holzforschung: 579-588, https://doi.org/10.1515/hf-2017-0130.

Zhan, T., Jiang, J., Lu, J., Zhang, Y., and Chang, J. (2018b). Influence of hygrothermal condition on dynamic viscoelasticity of Chinese fir (Cunninghamia lanceolata). Part 1: moisture adsorption. Holzforschung 72: 567-578. 